TRANSACTIONS OF THE

AMERICAN MATHEMATICAL SOCIETY

Volume 277, Number 2, June 1983

\title{
STRONGLY COHEN-MACAULAY SCHEMES AND RESIDUAL INTERSECTIONS
}

BY

CRAIG HUNEKE ${ }^{1}$

\begin{abstract}
ABSTRACr. This paper studies the local properties of closed subschemes $Y$ in Cohen-Macaulay schemes $X$ such that locally the defining ideal of $Y$ in $X$ has the property that its Koszul homology is Cohen-Macaulay. Whenever this occurs $Y$ is said to be strongly Cohen-Macaulay in $X$. This paper proves several facts about such embeddings, chiefly with reference to the residual intersections of $Y$ in $X$. The main result states that any residual intersection of $Y$ in $X$ is again Cohen-Macaulay.
\end{abstract}

Introduction. Our purpose in this paper is to investigate a property of a closed subscheme $Y$ in a Noetherian Cohen-Macaulay scheme $X$, which we call strong Cohen-Macaulayness. This is a local property which we first describe for a local ring. Let $X=\operatorname{Spec}(R)$, where $R$ is a Cohen-Macaulay local ring, and let $Y=\operatorname{Spec}(R / I)$ $(I=I(Y))$. Fix any (not necessarily minimal) generating set $f_{1}, \ldots, f_{n}$ of $I$. Let $H_{i}(f ; R)$ denote the $i$ th homology of the Koszul complex associated to $f_{1}, \ldots, f_{n}$. We define $Y$ to be strongly Cohen-Macaulay in $X$ if $H_{i}(f ; R)$ are either zero, or Cohen-Macaulay modules and if in addition $Y$ is generically a complete intersection. This last condition means that $I_{P}$ is generated by a regular sequence for every prime ideal $P$ which is minimal over $I$.

As $H_{0}(f ; R)=R / I, Y$ is necessarily Cohen-Macaulay if it is strongly CohenMacaulay in $X$.

If $X$ is any Noetherian Cohen-Macaulay scheme and $Y$ is any closed subscheme, then we say $Y$ is strongly Cohen-Macaulay in $X$ if this statement is true locally for every $y \in Y$.

The statement " $Y$ is strongly Cohen-Macaulay in $X$ " has several good geometric properties. First of all, this property does not depend upon the generating set of the defining ideal of $\vartheta_{Y, y}$ in $\vartheta_{X, y}$. If $X=\operatorname{Spec}\left(k\left[Z_{1}, \ldots, Z_{n}\right]\right)$ and $X^{\prime}=$ $\operatorname{Spec}\left(k\left[T_{1}, \ldots, T_{m}\right]\right)$ where $k$ is a field, and if $Y$ is embedded in both $X$ and $X^{\prime}$, then $Y$ is strongly Cohen-Macaulay in $X$ if and only if $Y$ is strongly Cohen-Macaulay in $X^{\prime}$. If $Y$ is strongly Cohen-Macaulay in $X=\operatorname{Spec}\left(k\left[Z_{1}, \ldots, Z_{n}\right]\right)$ and $Y^{\prime}$ is strongly Cohen-Macaulay in $X^{\prime}=\operatorname{Spec}\left(k\left[T_{1}, \ldots, T_{m}\right]\right)$, then $Y \times Y^{\prime}$ is strongly CohenMacaulay in $X \times X^{\prime}$ (Corollary 1.12).

The most obvious example of a strongly Cohen-Macaulay embedding is the case when $Y$ is a local complete intersection in $X$, i.e. the defining ideal of $Y$ in $X$ is

Received by the editors June 2, 1982.

1980 Mathematics Subject Classification. Primary $13 \mathrm{H} 10$.

'The author was supported by an NSF postdoctoral fellowship.

(C)1983 American Mathematical Society 0002-9947/82/0000-0707/\$07.00 
locally generated by a regular sequence. In this case, $Y$ is automatically CohenMacaulay (as $X$ is assumed to be), while locally, the higher Koszul homology of the defining ideal of $Y$ is zero (if we choose a minimal generating set for the ideal of $Y$ ).

We are motivated by three reasons to separate this class of subschemes from the general class of Cohen-Macaulay subschemes. First of all, strongly Cohen-Macaulay schemes have been recently studied by Simis and Vasconcelos [S-V] who are able to prove many properties concerning the blow-ups of these schemes and also are able to prove isomorphisms of the "residual scheme" of $Y$ and the blow-up of $Y$ (under suitable extra hypothesis). Recall the residual scheme of $Y$ is the scheme $\mathbf{P}(I(Y))$. (See Kleiman [K] for a discussion of these ideas.) In fact Simis and Vasconcelos are able to prove isomorphisms of the homogeneous coordinate rings of these two schemes under very general circumstances. We will discuss their work in detail in $\S \S 4$ and 5. (See also [Ku] for work concerning when the residual scheme and blow-up are isomorphic.)

The second reason for our interest arises from the fact that a general class of such schemes is found "in nature". To describe how these schemes are found we first recall the definition of liaison (see Peskine and Szpiro [P-S-1]).

Definition 0.1. Let $X$ be a Cohen-Macaulay Noetherian scheme, and let $V_{1}$ and $V_{2}$ be two closed subschemes of $X$. We say $V_{1}$ and $V_{2}$ are directly linked (written $V_{1}-V_{2}$ ) if there is a complete intersection $Z \subseteq V_{1} \cap V_{2}$ such that

(i) $\underline{I}\left(V_{2}\right) / \underline{I}(Z)=\operatorname{Hom}_{\Theta_{X}}\left(\Theta_{V_{1}}, \theta_{Z}\right)$, and

(ii) $\underline{I}\left(V_{1}\right) / \underline{I}(Z)=\operatorname{Hom}_{\theta_{x}}\left(\theta_{V_{2}}, \theta_{Z}\right)$.

Here $\underline{I}(Y)$ is the ideal sheaf of a closed subscheme $Y$ of $X$. If there exist $V_{1}, \ldots, V_{n}$ such that $V \smile V_{1} \smile \cdots \smile V_{n} \smile V^{\prime}$, we say $V$ and $V^{\prime}$ are linked in $X$ (written $V \sim V^{\prime}$ ).

The following theorem is proved in [Hu-1].

THEOREM 0.2. Let $X$ be a Gorenstein Noetherian scheme and suppose $Y \subseteq X$ is a closed subscheme. If $Y \sim Z$ where $Z$ is a complete intersection in $X$, then $Y$ is strongly Cohen-Macaulay in $X$.

For the benefit of those readers not conversant with liaison we pause to note two examples of such schemes.

Proposition 0.3. Let $X=\operatorname{Spec}(R)$ where $R$ is a regular local ring. Suppose $Y \subseteq X$ is a Cohen-Macaulay subscheme. If either

(i) $\operatorname{codim} Y=2$, or

(ii) codim $Y=3$ and $Y$ is Gorenstein,

then $Y$ is strongly Cohen-Macaulay in $X$.

For in either case $Y \sim Z$, a complete intersection in $X$. In the situation (i), this latter fact was proved by Peskine and Szpiro [P-S-1], while in case (ii) this fact was proved by $\mathbf{J}$. Watanabe [W].

We will later give some more examples of the type given in Theorem 0.2.

The third reason for separating this class of subschemes is the main reason for our interest in them. Namely, such schemes have very strong residual intersection properties. This author was surprised that these schemes could have such strong 
properties. The description of the residual intersection properties enjoyed by strongly Cohen-Macaulay schemes is the main result of this paper. We describe the main theorem in detail here.

Let $X=\operatorname{Spec}(R)$, where $R$ is a Cohen-Macaulay local ring and let $Y=V(I) \subset X$ be a closed subscheme of $X$. Let $C_{k}(Y)=C_{k} \subset X$ be the set of points $x \in X$ such that $v\left(I_{x}\right) \geqslant k$, where $v(-)$ denotes the least number of generators. We let

$$
c_{1}=c_{1}(Y)=\operatorname{codim}\left(Y \cap C_{1}(Y)\right), \quad c_{i}=c_{i}(V)=\operatorname{codim}\left(C_{i}(Y)\right) \quad \text { if } i>1 .
$$

The codimension of $\varnothing$ is by definition infinity.

Definition 0.4 [A-N, p. 312]. We say $Y$ satisfies condition $G_{s}$ if $c_{i} \geqslant i$ for $i=1, \ldots, s$.

REMARK 0.5 . The scheme $Y \subset X$ satisfies $G_{s}$ if and only if $v\left(I(Y)_{y}\right) \leqslant \operatorname{codim} \overline{\{y\}}$ for all $y \in Y$ such that $\operatorname{codim} \overline{\{y\}} \leqslant s-1$.

We prove the above statement as follows. Assume $Y$ satisfies $G_{s}$ and let $y \in Y$, $k=\operatorname{codim} \overline{\{y\}} \leqslant s-1$. If $v\left(I_{y}\right)>k$, then $y \in C_{k+1}(Y)$. Since $\overline{\{y\}} \subseteq C_{k+1}(Y)$ (this is a closed set), codim $\overline{\{y\}} \geqslant k+1$, contradicting our assumption.

Conversely, suppose $v\left(I_{y}\right) \leqslant \operatorname{codim} \overline{\{y\}}$ for all $y \in Y$ such that codim $\overline{\{y\}} \leqslant s-1$, and yet $c_{i}<i$ for some $i \leqslant s$. This inequality implies there is a $y \in Y$ such that $v\left(I_{y}\right) \geqslant i$ but $\operatorname{codim}\{\bar{y}\}<i$, which clearly contradicts our assumption. This finishes the proof of Remark 0.5 .

In particular we observe that the following three conditions are equivalent (set $n=v(I))$ :

(1) $Y$ satisfies $G_{\infty}$

(2) $v\left(I_{y}\right) \leqslant \operatorname{codim} \overline{\{y\}}$ for all $y \in Y$.

(3) $Y$ satisfies $G_{n}$.

Our main result can now be stated.

THEOREM 3.1. Let $Y$ be a strongly Cohen-Macaulay closed subscheme of a CohenMacaulay local scheme $X=\operatorname{Spec}(R)$. Put $k=\operatorname{codim}_{X} Y$. Let $I=$ the ideal defining $Y$. Suppose $s>k$ is an integer and let $A=\left(a_{1}, \ldots, a_{s}\right)$ be an ideal such that $A_{y}=I_{y}$ if $y \in Y$ and $\operatorname{codim} \overline{\{y\}} \leqslant$ s. Set $J=(A: I), Z=V(J)$. If $Y$ satisfies $G_{s}$, then:

(i) $Z$ is Cohen-Macaulay, $\operatorname{codim} Z=s$.

(ii) $Z \cup Y=V(A)$ (scheme-theoretically).

(iii) $Z \cap Y$ is Cohen-Macaulay of codimension $s+1$.

The subscheme $Z$ is called a residual intersection of $Y$ in $X$.

The interested reader should compare this result with Theorem 2.1, p. 312 of [A-N]. In their paper, Artin and Nagata are interested in proving this theorem in the case that $Y$ is a complete intersection in $X$. However, their theorem is stated too generally. In this general form, the result is incorrect. We give a counterexample (Example 3.3) to the statement of Theorem 2.1 of [A-N]. In fact, a subscheme $Y$ of $X$ which satisfies the conclusion of Theorem 3.1 is very close to being strongly Cohen-Macaulay. In the last section we are able to prove a partial converse.

THEOREM 5.1. Suppose $X$ is a Gorenstein affine scheme and $Y \subseteq X$ is a closed subscheme which satisfies $G_{\infty}$. In addition assume $c_{i}(Y) \geqslant i+2$ if $i \geqslant \operatorname{codim} Y+3$. 
Assume that the conclusion of Theorem 3.1 holds for any residual intersection $Z$ of $Y$. Then $Y$ is strongly Cohen-Macaulay in $X$.

The proof is obtained by comparing such schemes to those whose defining ideals are generated by $d$-sequences (see the last section for details). The proof shows that there are many large classes of strongly Cohen-Macaulay subschemes.

Theorem 3.1 has several interesting applications which we discuss in $\S 4$. Let $V$ be an $r$-dimensional vector space over a field $k$ and let $W$ be an $s$-dimensional vector space over $k$. Let $D_{r, s}$ be the variety of maps in $\operatorname{Hom}(V, W)$ of rank at most $r-1$. It is well known that

$$
D_{r, s}=\operatorname{Spec}\left(k\left[X_{i j}\right] /\left(\sqrt{I_{r}}(X)\right)\right.
$$

where $X=\left(x_{i j}\right)$ is an $r \times s$ generic matrix. It is known that $D_{r, s}$ is Cohen-Macaulay and further that $\sqrt{I}_{r}(X)=I_{r}(X)$. We show how the Cohen-Macaulayness of $D_{r, s}$ follows from the strong Cohen-Macaulayness of $D_{r, r+1}$. In fact, $D_{r, r+1}$ is a strong Cohen-Macaulay subscheme of $\mathbf{A}_{k}^{r(r+1)}$, and $D_{r, r+1}$ satisfies $G_{\infty}$. Further, the scheme $D_{s-r+1, r}$ is a residual intersection of $D_{r, r+1}$. By Theorem 3.1, $D_{s-r+1, r}$ is CohenMacaulay! This example illustrates the theme of this paper; a strongly CohenMacaulay scheme $Y$ in $X$ inherently contains a great deal of information about schemes of lower dimension in $X$, namely those which are residual intersections of $Y$.

Another application is a new proof of the theorem of Simis and Vasconcelos [S-V] which we have already referred to in this introduction. We review this result. Let $X=\operatorname{Spec}(R)$ where $R$ is a Cohen-Macaulay local ring and let $Y \subseteq X$ be a closed subscheme, $Y=V(I)$. By $G(Y)$ denote the scheme $\operatorname{Spec}\left(\bigoplus_{n \geqslant 0} I^{n} / I^{n+1}\right)$. Set $n=$ $v(I), I=\left(a_{1}, \ldots, a_{n}\right)$, and let $\tilde{Y}=\operatorname{Spec}\left(R\left[T_{1}, \ldots, T_{n}, S\right] / J\right)$ where $J$ is the ideal generated by $S T_{i}-a_{i}$, and all the polynomials $\sum_{i=1}^{n} b_{i} T_{i}$ such that $\Sigma b_{i} a_{i}=0$. Of course, $\tilde{Y}$ is closely related to the scheme $\operatorname{Spec}(\operatorname{Sym} I)$. Let $R(Y)$ be $\operatorname{Spec}\left(\oplus_{n \in \mathbf{Z}} I^{n}\right)$ where $I^{n}=R$ if $n \leqslant 0$. It is clear that there is a closed immersion of $R(Y)$ into $\tilde{Y}$. An important question is when $\tilde{Y}=R(Y)$. Whenever this occurs, $\operatorname{Sym}(I)=\bigoplus_{n \geqslant 0} I^{n}$. If $R(Y)=\tilde{Y}$, then it is well known that $Y$ must satisfy $G_{\infty}$. If $Y$ is strongly Cohen-Macaulay in $X$, Simis and Vasconcelos show the opposite also holds. (In fact they are also able to show the symmetric algebra of $I$ and the Rees algebra of $I$ are isomorphic and Cohen-Macaulay.)

TheOREM 0.6 [S-V]. Let $X$ be as above, and $Y \subseteq X$ a strongly Cohen-Macaulay closed subscheme of $X$. Suppose $Y$ satisfies $G_{\infty}$. Then

(i) $\tilde{Y}=R(Y)$, and

(ii) $\tilde{Y}$ is Cohen-Macaulay.

Set $n=v(I(Y))$ and let $T=\operatorname{Spec}\left(R\left[T_{1}, \ldots, T_{n}, S\right]\right)$. Then $R(Y) \subset \tilde{Y} \subset T$, where these are all closed immersions. Let $Y^{\prime}=V((I, S))$ in $T$. Then $Y^{\prime}$ is strongly Cohen-Macaulay in $T$ if $Y$ is so in $X$, and $Y^{\prime}$ also will satisfy $G_{\infty}$ if $Y$ does. We will show that $\tilde{Y}$ is always a residual intersection of $Y^{\prime}$. Under these conditions, Theorem 3.1 shows $\tilde{Y}$ is Cohen-Macaulay. Further the condition (ii) of Theorem 3.1 in this case is equivalent to the assertion that $\tilde{Y}=R(Y)$. 
One of the key ingredients of Theorem 3.1 is Proposition 1.6, which shows that strongly Cohen-Macaulay subschemes of Cohen-Macaulay schemes have a linkage property which in general is not held by Cohen-Macaulay ideals. Namely,

Proposition 1.6. Let $R$ be a Cohen-Macaulay local ring and I a strongly CohenMacaulay ideal of $R$. Suppose $J$ is linked to $I$. Then $R / J$ is Cohen-Macaulay.

This proposition is false if we only assume $R / I$ to be Cohen-Macaulay [P-S-1].

As we mentioned above, the last section of the paper is devoted to relating strong Cohen-Macaulayness to $d$-sequences. We refer the reader to this section for the relevant definitions. Of particular interest is Theorem 5.9, which gives a usable criterion to determine if a scheme $Y$ is strongly Cohen-Macaulay.

1. Preliminaries. In this section we present the basic facts concerning Koszul homology, and prove several basic propositions we will need in the later sections. Our work is entirely local in nature in this section. Throughout this section we fix a Cohen-Macaulay local ring $R$. If $I=\left(f_{1}, \ldots, f_{n}\right)$ is an ideal of $R$, the Koszul complex of $f$, denoted $K .(f ; R)$ is the tensor product of the complexes, $0 \rightarrow R \stackrel{f_{i}}{\rightarrow} R \rightarrow 0$. (This complex is in degrees 0 and 1.)

We denote the $i$ th homology of this complex by $H_{i}(f ; R)$. Thus $H_{0}(f ; R)=R / I$. If $M$ is any $R$-module, by $H_{i}(f ; M)$ we denote the $i$ th homology of the complex $K .(f ; R) \otimes M$.

The homology $H_{i}(f ; M)$ depends upon the generating set $f_{1}, \ldots, f_{n}$ of $I$. In general, we have the formula

$$
H_{i}(\underline{f}, 0 ; M)=H_{i}(\underline{f} ; M) \oplus H_{i-1}(\underline{f} ; M) .
$$

In addition, the Koszul homology of $M$ with respect to any two minimal generating sets of $I$ is isomorphic. The following remark is immediate from (1.1).

ReMarK 1.2. The property " $H_{i}(f ; M)$ is Cohen-Macaulay for all $0 \leqslant i \leqslant m$ " is independent of the generating set $f_{1}, \ldots, f_{n}$ of $I$. In particular the property " $H_{i}(f ; M)$ is Cohen-Macaulay for all $i$ " is independent of the generating set of $I$.

Recall that if depth $I M=k$, and $\left(f_{1}, \ldots, f_{n}\right)=I$, then $H_{j}(f ; M)=0$ for $j>n-k$. In addition, $H_{n-k}(f ; M) \simeq \operatorname{Hom}\left(M / I M, R /\left(f_{1}, \ldots, f_{k}\right)\right)$ if $f_{1}, \ldots, f_{k}$ is a regular $M$-sequence. In particular if $M=R$ and $R$ is Gorenstein, $H_{n-k}(f ; R) \simeq K_{R / I}$, the canonical module of $R / I$.

Remark 1.3 (SEE [Hu-2]). If $R$ is Cohen-Macaulay local and $I$ is an ideal of $R$, $I=\left(f_{1}, \ldots, f_{n}\right)$, then $\operatorname{dim} H_{j}(f ; R)=\operatorname{dim} R / I$ for all $j$ such that $H_{j}(f ; R) \neq 0$.

There are two fundamental exact sequences which we will use throughout this paper.

LEMMA 1.4. Let $I$ be an ideal of a ring $R, I=\left(f_{1}, \ldots, f_{n}\right)$.

(1) If $f_{1}$ is a regular element of $R$, then there is an exact sequence,

$$
0 \rightarrow H_{i}(\underline{f} ; R) \rightarrow H_{i}\left(\underline{f} ; R / f_{1} R\right) \rightarrow H_{i-1}(\underline{f} ; R) \rightarrow 0 .
$$


(2) Suppose $(0: I) \cap I=(0)$. Then there is an exact sequence

$$
0 \rightarrow K \rightarrow H_{i}(\underset{f}{f} R) \rightarrow H_{i}(\underline{f} ; R /(0: I)) \rightarrow 0
$$

for $i \geqslant 0$, where $K$ is isomorphic to a direct sum of copies of $(0: I)$.

Proof. For (1) we use the exact sequence

$$
0 \rightarrow R \stackrel{f_{1}}{\rightarrow} R \rightarrow R / f_{1} R \rightarrow 0 .
$$

This gives a long exact sequence for the Koszul homology, and since $f_{1} H_{i}(f ; M)=0$ for any $R$-module $M$, this long exact sequence breaks up into the short exact sequences of (1).

The exact sequences of (2) follow similarly from the exact sequence

$$
0 \rightarrow(0: I) \rightarrow R \rightarrow R /(0: I) \rightarrow 0 .
$$

We get a long exact sequence on Koszul homology,

$$
\begin{aligned}
\cdots & \rightarrow H_{i+1}(\underline{f} ; R) \rightarrow H_{i+1}(\underline{f} ; R /(0: I)) \rightarrow H_{i}(\underline{f} ;(0: I)) \\
& \rightarrow H_{i}(\underline{f} ; R) \rightarrow H_{i}(\underline{f} ; R /(0: I)) \rightarrow \cdots .
\end{aligned}
$$

Assertion (2) follows from two claims. First, since $I$ annihilates $(0: I), H_{i}(f ;(0: I))$ $=(0: I)^{\left(i_{i}^{n}\right)}$. Secondly, we claim all the maps $H_{k}(\underset{-}{f} ; R) \stackrel{i}{\rightarrow} H_{k}(f ; R /(0: I))$ are surjective.

Suppose $\bar{\alpha}$ is a cycle which represents a class in $H_{k}(f ; R /(0: I))$. The map $i$ is just the restriction of a cycle in $H_{k}(f ; R)$ to $H_{k}(f ; R /(0: \bar{I}))$. Let $\alpha$ be any lifting of $\bar{\alpha}$ to $\Lambda^{k} F$, where $F$ is a free module on $n$-generators, and we have identified the Koszul complex with $\Lambda F$. We denote the differential by $d$. Then the fact that $\bar{\alpha}$ is a cycle implies $d \alpha \in(0: I)\left(\Lambda^{k-1} F\right)$. In addition, by definition of the differential $d, d \alpha \in$ $I\left(\Lambda^{k-1} F\right)$. As $\Lambda^{k-1} F$ is a free $R$-module and $(0: I) \cap I=0, d \alpha=0$ and so $\alpha$ represents a class in $H_{k}(f ; R)$. It follows $i$ is onto.

Corollary 1.5. Let $I$ be an ideal of the Cohen-Macaulay local ring $R$. Suppose $I=\left(x_{1}, \ldots, x_{n}\right)$ with $x_{1}$ a regular element of $R$. Let "'" denote the homomorphism from $R$ to $R / R x_{1}$. Then $I$ is strongly Cohen-Macaulay in $R$ if and only if $I^{\prime}$ is strongly Cohen-Macaulay in $R^{\prime}$.

Proof. This corollary follows at once from the exact sequence of Lemma 1.4(1) and from Remark 1.3.

We now prove the important proposition (see [HU-2]).

Proposition 1.6. Let $R$ be a Cohen-Macaulay local ring and let I be an ideal of $R$. Suppose I is strongly Cohen-Macaulay. Let $x_{1}, \ldots, x_{k}$ be a maximal $R$-sequence inside $I$, and place $J=(\underline{x}: I)$. Then $R / J$ is Cohen-Macaulay.

Proof. Extend $x_{1}, \ldots, x_{k}$ to a generating set $x_{1}, \ldots, x_{n}$ of $I$. By Corollary 1.5, we may reduce modulo $x_{1}, \ldots, x_{k}$ and assume that $J=(0: I)$.

We induct on $\operatorname{dim} R=\operatorname{dim} R / I$ to prove the proposition. If $\operatorname{dim} R=0$, there is nothing to prove. Otherwise, we may choose a $z$ in $R$ such that $z$ is not a zero-divisor 
on $R$, or on $H_{j}(\underline{x} ; R)$ for all $j$ such that $j \leqslant n$. (Here we relabel a generating set of $I=\left(x_{1}, \ldots, x_{n}\right)$.)

Using the long exact sequence of Koszul homology determined by the short exact sequence $0 \rightarrow R \stackrel{z}{\rightarrow} R \rightarrow R / z R \rightarrow 0$, we obtain exact sequences,

$$
0 \rightarrow H_{i}(\underline{x} ; R) \stackrel{z}{\rightarrow} H_{i}(\underline{x} ; R) \rightarrow H_{i}(\underline{x} ; R / z R) \rightarrow 0 .
$$

Further if we denote the homomorphism from $R$ to $R / z R$ by “' ", we see from (1) that because $I$ is strongly Cohen-Macaulay in $R, I^{\prime}$ must be strongly Cohen-Macaulay in $R^{\prime}$. Since $\operatorname{dim} R^{\prime}=\operatorname{dim} R-1<\operatorname{dim} R$, by induction $R^{\prime} /\left(0: I^{\prime}\right)$ is CohenMacaulay. Lifting back to $R$, we see that $R^{\prime} /\left(0: I^{\prime}\right)=R /(z:(I, z))$ is CohenMacaulay. Since $z$ is not a zero-divisor in $r, z$ is not a zero-divisor on $R /(0: I)$. Consequently, $R /((0: I), z)$ is Cohen-Macaulay if and only if $R /(0: I)$ is so. We will finish this proof by establishing the claim

$$
((0: I), z)=(z:(I, z)) .
$$

The remarks at the beginning to this section show that $H_{n}(\underline{x} ; R)=(0: I)$, while $H_{n}\left(\underline{x} ; R^{\prime}\right)=H_{n}\left(\underline{x}^{\prime} ; R^{\prime}\right)=\left(0: I^{\prime}\right)$. The exact sequences $(1)$ show that $(0: I) / z(0: I)$ $\rightarrow\left(0: I^{\prime}\right)$ is an isomorphism. The map is the obvious one, and takes an element $s$ of $(0: I)$ to $s^{\prime} \in\left(0: I^{\prime}\right)$.

We obtain

$$
\frac{((0: I), z)}{(z)} \stackrel{\sim}{\rightarrow} \frac{(z:(I, z))}{(z)} .
$$

This isomorphism proves the claim, and hence proves the proposition.

We remark that the proposition is false if one only assumes $R / I$ to be CohenMacaulay. See Example 1.8 in [P-S-1]. This proposition and the next give us the main tools needed in the induction to prove Theorem 3.1.

Proposition 1.7 (SEe [Hu-2]). Let $R$ be a Cohen-Macaulay local ring and let I be a strongly Cohen-Macaulay ideal of $R$. Suppose $x_{1}, \ldots, x_{k}$ are a maximal regular sequence contained in $I$, and let $J=\left(x_{1}, \ldots, x_{k}: I\right)$. If

(1) $J \cap I=\left(x_{1}, \ldots, x_{k}\right)$, and

(2) $\mathrm{ht}(J+I) \geqslant k+1$,

then $I^{\prime}$ is strongly Cohen-Macaulay in $R^{\prime}$, where " " denotes the homomorphism from $R$ to $R / J$.

Proof. By Corollary 1.5 , we may assume $k=0$, i.e. $J=(0: I)$. Let $f_{1}, \ldots, f_{n}$ generate $I$. By Proposition 1.6, $R / J$ is Cohen-Macaulay; in particular by Remark 1.3, $\operatorname{dim} H_{j}\left(f^{\prime} ; R\right)=\operatorname{dim} R^{\prime} / I^{\prime}=\operatorname{dim} R / I+J<\operatorname{dim} R / J$ by assumption (2) of the proposition.

Set $d=\operatorname{dim} R / I=\operatorname{dim} R / J=\operatorname{dim} R$. It is enough to show depth $H_{j}\left(f^{\prime} ; R^{\prime}\right) \geqslant$ $d-1$. The exact sequence $0 \rightarrow J \rightarrow R \rightarrow R^{\prime} \rightarrow 0$, together with Proposition 1.6, show that depth $J=$ depth $R=d$. From Lemma 1.4(2), there are exact sequences

$$
0 \rightarrow K \rightarrow H_{i}(\underline{f} ; R) \rightarrow H_{i}\left(\underline{f}^{\prime} ; R^{\prime}\right) \rightarrow 0
$$

for every $i \geqslant 0$. Since $K$ is isomorphic to a direct sum of copies of $J$, depth $K=d$. By assumption depth $H_{i}(f ; R)=d$. It easily follows that depth $H_{i}\left(f_{-}^{\prime} ; R^{\prime}\right) \geqslant d-1$. 
We remark that assumption (2) of Proposition 1.7 follows from assumption (1) provided $R_{P}$ is Gorenstein for all prime ideals $P$ such that $P$ is minimal over $I$.

REMARK 1.8. We have seen that if $z$ is a nonzero-divisor on $R / I$ and $R$ $\left(I=\left(f_{1}, \ldots, f_{n}\right)\right)$, then

$$
H_{i}(\underline{f}, z ; R) \simeq H_{i}(\underline{f} ; R) \otimes_{R} R / R z .
$$

It easily follows that each of the following statements implies the other two. Let $z_{1}, \ldots, z_{k}$ be a regular sequence on $R$ and on $R / I$.

(i) $I$ is strongly Cohen-Macaulay in $R$.

(ii) $\left(I, z_{1}, \ldots, z_{k}\right)$ is strongly Cohen-Macaulay in $R$.

(iii) $I^{\prime}$ is strongly Cohen-Macaulay in $R^{\prime}$, where $R^{\prime}=R /\left(z_{1}, \ldots, z_{k}\right)$.

Suppose $\operatorname{Spec}(T)$ is embedded in the spectrum of two different polynomial rings, $R_{1}=k\left[T_{1}, \ldots, T_{n}\right]$ and $R_{2}=k\left[Y_{1}, \ldots, Y_{m}\right]$, so that there is an isomorphism, $R_{1} / I_{1} \simeq$ $T \simeq R_{2} / I_{2}$.

We claim $I_{1}$ is strongly Cohen-Macaulay in $R_{1}$ if and only if $I_{2}$ is strongly Cohen-Macaulay in $R_{2}$. Denote the tensor product $R_{1} \otimes_{k} R_{2}$ by $S$, and let $\pi$ be the isomorphism $R_{1} / I_{1} \rightarrow R_{2} / I_{2}$. It easily follows that in $S$

$$
\left(I_{1} S, T_{i}-\pi\left(T_{i}\right)\right)=\left(I_{2} S, Y_{j}-\pi^{-1}\left(Y_{j}\right)\right)
$$

and that $\left\{T_{i}-\pi\left(T_{i}\right)\right\}$ form a regular sequence on $S / I_{1} S$ and $S$ and that $Y_{j}-\pi^{-1}\left(Y_{j}\right)$ form a regular sequence on $S / I_{2} S$ and $S$. The above equivalences now imply our claim.

Next we wish to discuss how strong Cohen-Macaulayness behaves under products. Our goal is to prove

THEOREM 1.9. Let $R$ be a regular local ring, and $I$ and $J$ two ideals of $R$ such that $Y=\operatorname{Spec}(R / I)$ and $Z=\operatorname{Spec}(R / J)$ are strongly Cohen-Macaulay in $X=\operatorname{Spec}(R)$. Assume $I \cap J=I J$. Then $Y \cap Z$ is strongly Cohen-Macaulay in $X$.

Although this theorem is essentially the Künneth formula, there are several details which must be checked. We separate some of these details in two lemmas.

LEMMA 1.10. Suppose $R$ is a regular local ring, and $I$ and $J$ are two ideals of $R$ such that $R / I, R / J$ are Cohen-Macaulay and $I \cap J=I J$. If $M$ is a finite maximal Cohen-Macaulay $R / I$-module and $N$ is a finite maximal Cohen-Macaulay $R / J$ module, then $\operatorname{Tor}_{i}^{R}(M, N)=0$ for all $i \geqslant 1$. Further $M \otimes_{R} N$ is a maximal CohenMacaulay module of $R /(I+J)$.

Proof. Our assumption reads, $\operatorname{Tor}_{1}^{R}(R / I, R / J)=0$. By rigidity $[\mathbf{L}]$,

$$
\operatorname{Tor}_{i}^{R}(R / I, R / J)=0 \text { for all } i \geqslant 1 \text {. }
$$

Let

$$
F .: 0 \rightarrow F_{k} \stackrel{f_{k}}{\rightarrow} \cdots \stackrel{f_{1}}{\rightarrow} F_{0} \rightarrow M \rightarrow 0
$$

be a free resolution for $M$ over $R$. Here $k=$ ht $I$. To show $F \otimes N$ is exact, it is enough by the Buchsbaum-Eisenbud criterion [B-E] to prove $\operatorname{grade}_{I\left(f_{j}\right)} N \geqslant j$, where $I\left(f_{j}\right)$ is the ideal generated by the maximal nonvanishing minors of the map $f_{j}$. 
However, as $M$ is perfect, $\sqrt{I}=\sqrt{I}\left(f_{j}\right)$ for all $j, 1 \leqslant j \leqslant k$. Hence we must show $\operatorname{grade}_{I} N \geqslant k$. As $N$ is a maximal Cohen-Macaulay module for $R / J$, it is enough to prove grade ${ }_{I} R / J \geqslant k$. However, if we take a free resolution for $R / I$ and tensor it with $R / J$, the condition $\operatorname{Tor}_{i}(R / I, R / J)=0, i \geqslant 1$, forces $\operatorname{grade}_{I} R / J \geqslant k$.

Next we prove the last statement of Lemma 1.10. Let $F$. be a free resolution of $M$ over $R$ and let $G$. be a free resolution of $N$ over $R$. The tensor product $F \otimes G$. furnishes a free resolution of $M \otimes_{R} N$ since $\operatorname{Tor}_{i}^{R}(M, N)=0$ by the above argument. The length of this resolution is ht $I+\mathrm{ht} J=\mathrm{ht}(I+J)$ (by the assumption $\left.\operatorname{Tor}_{i}^{R}(R / I, R / J)=0\right)$. This proves $\operatorname{depth} M \otimes_{R} N=\operatorname{dim} R-\operatorname{ht}(I+J)=$ $\operatorname{dim} R / I+J$.

LEMMA 1.11. Let $F$. be a finite free complex over a Cohen-Macaulay local ring $R$ and suppose $M$ is an $R$-module with $\operatorname{Tor}_{i}\left(M, H_{j}\left(F_{0}\right)\right)=0$ for all $i \geqslant 1$ and all $j \geqslant 0$. Let $Z_{n}=n$th cycles of $F$. and $B_{n}=n$th boundaries. Then $\operatorname{Tor}_{i}\left(M, Z_{n}\right)=0=\operatorname{Tor}_{i}\left(M, B_{n}\right)$ for all $i \geqslant 1$ and all $n$.

Proof. $Z_{n}=\operatorname{ker}\left(F_{n} \rightarrow F_{n-1}\right)$, and $B_{n}=\operatorname{im}\left(F_{n+1} \rightarrow F_{n}\right)$. There are exact sequences

$$
\begin{aligned}
& 0 \rightarrow B_{n} \rightarrow Z_{n} \rightarrow H_{n} \rightarrow 0, \quad n \geqslant 0, \\
& 0 \rightarrow Z_{n} \rightarrow F_{n} \rightarrow B_{n-1} \rightarrow 0, \quad n \geqslant 1,
\end{aligned}
$$

where $H_{n}=H_{n}\left(F_{\text {. }}\right)$.

We induct on $n$ to show $\operatorname{Tor}_{i}\left(M, Z_{n}\right)=\operatorname{Tor}_{i}\left(M, B_{n}\right)=0$ for all $i$. If $n=0$, there is an exact sequence as in (1), $0 \rightarrow B_{0} \rightarrow Z_{0} \rightarrow H_{0} \rightarrow 0$. Since $Z_{0}=F_{0}$ and $\operatorname{Tor}_{i}\left(M, F_{0}\right)=\operatorname{Tor}_{i}\left(M, H_{0}\right)=0$ for all $i \geqslant 1$, it easily follows $\operatorname{Tor}_{i}\left(M, B_{0}\right)=0$ for all $i \geqslant 1$.

Assume $n>0$ and we have shown our claim for $n-1$. Then the vanishing of Tor for $B_{n-1}$ and $F_{n}$ implies $\operatorname{Tor}_{i}\left(M, Z_{n}\right)=0$ for all $i \geqslant 1$ from the exact sequence (2): now the vanishing for $H_{n}$ and $Z_{n}$ give $\operatorname{Tor}_{i}\left(M, B_{n}\right)=0$ for all $i \geqslant 1$ by the exact sequence (1).

We are now able to prove Theorem 1.9. We just copy the proof of the Künneth formula found in [M, pp. 166-168]; however the assumptions are too strong in the above theorem for our case so we must make some modifications. Rather than repeat the proof we simply note the salient points. First of all, our assumption that $\operatorname{Tor}_{1}^{R}(R / I, R / J)=0$ forces $\operatorname{Tor}_{i}^{R}(R / I, R / J)=0$ for all $i \geqslant 1$ by rigidity. By hypothesis, $R / I$ and $R / J$ are strongly Cohen-Macaulay in $R$; hence $H_{i}(I ; R)$ and $H_{i}(J ; R)$ are maximal Cohen-Macaulay modules (or zero) for $R / I$ and $R / J$ respectively. (Here by $H_{i}(I ; R)$ we mean fix some (any) generating set for $I$ and take Koszul homology.) Lemma 1.10 now implies $\operatorname{Tor}_{i}^{R}\left(H_{j}(I ; R), H_{k}(J ; R)\right)=0$ for all $i \geqslant 1, j \geqslant 0, k \geqslant 0$.

Let $K$. be the Koszul complex for $I$ (with respect to a fixed generating set) and let $L$. be the Koszul complex for $J$. Let $C_{n}=C_{n}\left(K\right.$.) be the cycles of $K$. and $D_{n}=$ $B_{n-1}(K)$ be the boundaries of $K$. By Lemma 1.11, $\operatorname{Tor}_{i}\left(C_{n}, H_{k}(J ; R)\right)=$ $\operatorname{Tor}_{i}\left(D_{n}, H_{k}(J ; R)\right)$ for all $i \geqslant 1, k \geqslant 0$, and $n \geqslant 0$. 
There is a short exact sequence of complexes, $0 \rightarrow C . \rightarrow K . \rightarrow D_{.} \rightarrow 0$, which remain exact after tensoring with $L$. . To continue the proof as in $[\mathbf{M}]$, we need to show $\operatorname{Tor}_{1}\left(C_{m}, H_{q}(L).\right)=0$, which we have shown, and we need to observe that

$$
H_{n}\left(L_{.}\right) \otimes D_{m}=H_{n}\left(L . \otimes D_{m}\right) \text {. }
$$

The $n$th homology of $L$. is described by the commutative diagram

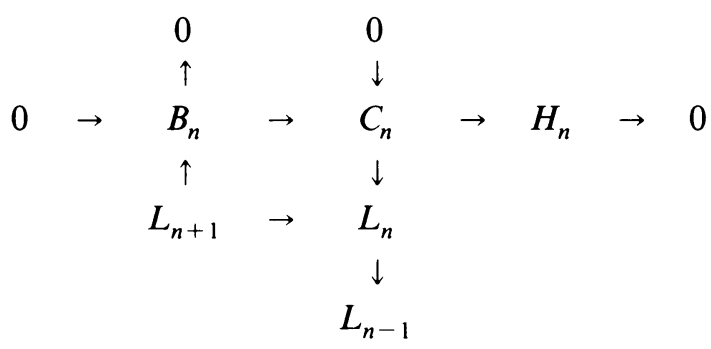

(see [M, Lemma 10.3, p. 167]).

Tensor this diagram with $D_{m}$. Since $\operatorname{Tor}_{1}\left(D_{m}, H_{n}\left(L_{.}\right)\right)=0$ by above, the middle row stays exact. $L_{n+1} \otimes D_{m} \rightarrow B_{n} \otimes D_{m}$ remains onto since tensor is right exact. Finally the middle column exact sequence gives a short exact sequence $0 \rightarrow C_{n} \rightarrow L_{n}$ $\rightarrow B_{n-1} \rightarrow 0$. If $\operatorname{Tor}_{1}\left(D_{m}, B_{n-1}\right)=0$ then the sequence

$$
0 \rightarrow C_{n} \otimes D_{n} \rightarrow L_{n} \otimes D_{m} \rightarrow L_{n-1} \otimes D_{m}
$$

will remain exact. Since we have shown $\operatorname{Tor}_{i}\left(D_{m}, H_{q}\left(L_{.}\right)\right)=0$, we may apply Lemma 1.11 to conclude $\operatorname{Tor}_{1}\left(D_{m}, B_{n-1}\right)=0$. This finishes the outline of the proof of the Künneth formula. We conclude,

$$
\sum_{m+q=n} H_{m}(I ; R) \otimes H_{q}(J ; R)=H_{n}(I+J ; R) .
$$

Here note the homology of $K . \otimes L$. is precisely $H_{n}(I+J ; R)$ while we have shown $\operatorname{Tor}_{1}^{R}\left(H_{m}\left(K_{.}\right), H_{q}\left(L_{.}\right)\right)=0$.

However by Lemma $1.10, H_{m}(I ; R) \otimes H_{q}(J ; R)$ are maximal Cohen-Macaulay modules for $R / I+J$. It now follows that $\operatorname{Spec}(R / I+J)$ is strongly CohenMacaulay in $\operatorname{Spec}(R)$.

Corollary 1.12. Let $X=\operatorname{Spec}\left(k\left[X_{1}, \ldots, X_{n}\right]\right)$ and $X^{\prime}=\operatorname{Spec}\left(k\left[T_{1}, \ldots, T_{m}\right]\right)$. Suppose $Y \subset X$ and $Y^{\prime} \subset X^{\prime}$ are strongly Cohen-Macaulay closed subschemes of $X$ and $X^{\prime}$ respectively. Then $Y \times Y^{\prime}$ is strongly Cohen-Macaulay in $X \times X^{\prime}$.

Proof. Let $I=I(Y), J=I\left(Y^{\prime}\right)$. Then $I\left(Y \times Y^{\prime}\right)$ is given by the ideal generated by $I+J$ in $k\left[X_{1}, \ldots, X_{n}, T_{1}, \ldots, T_{m}\right]=S$. If we show $I S \cap J S=I J S$, then we may apply Theorem 1.9. (As by definition we need only check strong Cohen-Macaulayness locally.) However, $I S \cap J S=I \cdot J S$ is clear.

2. Examples and remarks. In this section we present some known examples of strongly Cohen-Macaulay schemes and further give a simple criterion to decide if a scheme is not strongly Cohen-Macaulay. We also give some remarks concerning the duality of Koszul homology which we will later need. Finally we show that a conjecture of Vasconcelos is true for strongly Cohen-Macaulay schemes. Much of 
the first part of this section appears in [Hu-1]; therefore we do not give proofs for these results.

The largest known class of strongly Cohen-Macaulay subschemes comes from liaison as we noted in the introduction. As we stated there, this class includes perfect subschemes of codimension two in Cohen-Macaulay schemes and perfect Gorenstein schemes of codimension three in Gorenstein schemes. We list another such example here.

EXAMPLE 2.1. Let $X$ be a generic $n$ by $n+1$ matrix and $Y$ an $n+1$ by 1 matrix. J. Herzog [He-2] gave a resolution for the ideal $I_{1}(X Y)+I_{n}(X)=J$ in the polynomial ring $k[X, Y]=R$. In fact $R / J$ is Gorenstein of height $n+1$. It was shown in [Hu-1] that $J$ is in the linkage class of a complete intersection. Consequently $R / J$ is strongly Cohen-Macaulay in $R$.

Two other classes of examples are also known to be strongly Cohen-Macaulay.

Proposition 2.2. Let $R$ be a Cohen-Macaulay local ring, and $I$ an ideal of $R$ such that:

(i) $R / I$ is Cohen-Macaulay.

(ii) $I$ is generically a complete intersection.

(iii) $v(I)=\mathrm{ht} I+1$, where in general $v(M)$ is the least number of generators of the $R$-module $M$.

Then $R / I$ is strongly Cohen-Macaulay in $R$.

Proof. Let $I=\left(a_{1}, \ldots, a_{k}, a_{k+1}\right)$ where $a_{1}, \ldots, a_{k}$ is a regular sequence which generically generates $I$. From $\S 1$, we see that $H_{i}(\underline{a} ; R)=0$ for $i>1$, while $H_{0}(\underline{a} ; R)$ $=R / I$ is Cohen-Macaulay. Hence it remains to show $H_{1}(\underline{a} ; R)$ is Cohen-Macaulay. This module is isomorphic to $\left(a_{1}, \ldots, a_{k}: a_{k+1}\right) /\left(a_{1}, \ldots, a_{k}\right)$. Consider the exact sequence,

$$
\begin{aligned}
0 \rightarrow\left(\left(a_{1}, \ldots, a_{k}\right): a_{k+1}\right) /\left(a_{1}, \ldots, a_{k}\right) & \rightarrow R /\left(a_{1}, \ldots, a_{k}\right) \\
\rightarrow R /\left(a_{1}, \ldots, a_{k}: a_{k+1}\right) & \rightarrow 0 .
\end{aligned}
$$

To show the left-hand module of (1) is Cohen-Macaulay, it suffices to show the right-hand module is Cohen-Macaulay. (All these modules have the same dimension.) This fact, however, follows from the exact sequence

$$
0 \rightarrow R /\left(\left(a_{1}, \ldots, a_{k}\right): a_{k+1}\right) \rightarrow R /\left(a_{1}, \ldots, a_{k}\right) \rightarrow R /\left(a_{1}, \ldots, a_{k+1}\right) \rightarrow 0 .
$$

EXAMPLE 2.3. If $R$ is Gorenstein local and if $I$ is an ideal satisfying

(1) $v(I)=$ ht $I+2$,

(2) $R / I$ is Cohen-Macaulay,

then Avramov and Herzog [A-H] showed $R / I$ is strongly Cohen-Macaulay in $R$. In particular, if $\operatorname{dim} R / I=2$ and $R / I$ is normal, then $I$ is strongly Cohen-Macaulay if $v(I) \leqslant \operatorname{dim} R$. For example, the defining ideal $I$ of the surface $k\left[s^{8}, s^{5} t^{3}, s^{4} t^{4}, s^{3} t^{5}, t^{8}\right]$ is generated by $Z^{2}-X V, Y U-X V, Y^{3}-Z U X, X U^{2}-$ $Z Y^{2}$, and $U^{3}-Z Y V$. If $R=k[X, Y, Z, U, V]$, then $R / I$ is two dimensional normal and $v(I)=5 \leqslant \operatorname{dim} R$. Hence $R / I$ is strongly Cohen-Macaulay in $R$.

In [Hu-1] a criterion involving the module $T_{2}$ was given which gives a strong necessary condition for a scheme to be strongly Cohen-Macaulay. Let $R$ be as usual, 
and set $I=\left(f_{1}, \ldots, f_{n}\right)$. There is an exact sequence, $H_{1}(f ; R) \stackrel{\beta}{\rightarrow}(R / I)^{n} \stackrel{\alpha}{\rightarrow} I / I^{2} \rightarrow 0$. The maps $\alpha$ and $\beta$ are defined as follows: If $\left(\bar{r}_{1}, \ldots, \bar{r}_{n}\right) \in(R / I)^{n}, \alpha\left(\left(\bar{r}_{1}, \ldots, \bar{r}_{n}\right)\right)=$ $\sum r_{i} f_{i} \bmod I^{2}$. If $\left(s_{1}, \ldots, s_{n}\right)$ represents the class of an element in $H_{1}(f ; R)$ (that is, $\left.\sum_{i=1}^{n} s_{i} f_{i}=0\right)$, then $\beta\left(\left(\overline{s_{1}, \ldots, s_{n}}\right)\right)=\left(\bar{s}_{1}, \ldots, \bar{s}_{n}\right)$.

The kernel of $\beta$ is by definition $T_{2}^{X / Y}=T_{2}(X=\operatorname{Spec}(R), Y=\operatorname{Spec}(R / I))$.

The following proposition can be found in [Hu-2].

Proposition 2.4. Suppose $Y$ is strongly Cohen-Macaulay in $X$. Then $T_{2}^{X / Y}=0$.

Simis and Vasconcelos showed in [S-V] that $T_{2}$ also is equal to $\operatorname{ker}\left(\operatorname{Sym}_{2} I \rightarrow I^{2}\right)$. From this remark and from Proposition 2.4, the following example was given in [Hu-1].

EXAMPLE 2.5. Let $X$ be a generic $r \times s$ matrix $(r \leqslant s)$. Then $I_{t}(X)$ is not strongly Cohen-Macaulay if

(i) $1<t<r$, or

(ii) $t=r<s-1$.

In other cases, $I_{t}(X)$ is strongly Cohen-Macaulay.

Finally we wish to show how a conjecture of Vasconcelos is verified for strongly Cohen-Macaulay varieties (see [V]).

Proposition 2.6. Suppose $R$ is a local ring and $I$ an ideal such that $H_{i}(f ; R)$ are Cohen-Macaulay for all $i\left(\right.$ for some $(f)=I$ ). If $\operatorname{pd}_{R} I<\infty$ and $\operatorname{pd}_{R / I} I \bar{l} I^{2}<\infty$, then $I$ is generated by a regular sequence.

Proof. Notice that we do not need to assume $I$ is generically a complete intersection. In fact, if $P \supseteq I$ is a minimal prime of $I$, then all our assumptions still hold for $I_{P}$. In this case, since $\operatorname{dim}(R / I)_{P}=0$, and $\mathrm{pd} I_{P} / I_{P}^{2}<\infty, I_{P} / I_{P}^{2}$ is free. Now Vasconcelos has proved that $I_{P}$ is generated by a regular sequence. By Proposition $2.4, T_{2}=0$. Hence we have an exact sequence,

$$
0 \rightarrow H_{1}(\underline{f} ; R) \rightarrow(R / I)^{n} \rightarrow I / I^{2} \rightarrow 0 .
$$

We conclude that $\operatorname{pd}_{R / I} H_{1}(f ; R)<\infty$. As $H_{1}(f ; R)$ is by assumption CohenMacaulay, $H_{1}(f ; R)$ is free. Now a result of Gulliksen [G-L] shows $I$ is generated by a regular sequence.

Applying this proposition to the examples of this section recovers many of the results of [V].

Next we wish to investigate some duality which holds on the Koszul complex. In [He-1] the following remark was essentially proved: Suppose $R$ is a Gorenstein local ring and $I$ an ideal of $R, I=\left(a_{1}, \ldots, a_{n}\right)$. Set

$$
d(I)=d=\text { deviation of } I=n-\text { ht } I .
$$

If $H_{0}(\underline{a} ; R), \ldots, H_{j}(\underline{a} ; R)$ are Cohen-Macaulay modules, then so are $H_{d}(\underline{a} ; R)$, $\ldots, H_{d-j}(\underline{a} ; R)$ and

$$
H_{d-j}(\underline{a} ; R) \simeq \operatorname{Hom}\left(H_{i}(\underline{a} ; R), H_{d}(\underline{a} ; R)\right) .
$$

There is always a pairing $H_{i}(\underline{a} ; R) \times H_{d-i}(\underline{a} ; R) \rightarrow H_{d}(\underline{a} ; R)$ given by the exterior product on the Koszul homology. If $R$ is Gorenstein, $H_{d}(\underline{a} ; R) \simeq K_{R / I}$, the canonical module of $R / I$. 
Proposition 2.7. Suppose $H_{i}(\underline{a} ; R)$ are reflexive modules for every $i, 0 \leqslant i \leqslant d$. Then the pairing above is perfect, that is,

$$
H_{i}(\underline{a} ; R)=\operatorname{Hom}\left(H_{d-i}(\underline{a} ; R), H_{d}(\underline{a} ; R)\right)
$$

and

$$
H_{d-i}(\underline{a} ; R)=\operatorname{Hom}\left(H_{i}(\underline{a} ; R), H_{d}(\underline{a} ; R)\right) .
$$

Proof. We denote $H_{j}(\underline{a} ; R)$ by $H_{j}$ for simplicity, and we only show the first equality. From the pairing, there is a map, $H_{i} \stackrel{f}{\rightarrow} \operatorname{Hom}\left(H_{d-i}, H_{d}\right)$. By assumption, both of these modules are reflexive and so it is enough to show $f$ is an isomorphism in codimension at most one. In this case the modules $\left(H_{i}\right)_{p}$ are Cohen-Macaulay modules and we may apply the result of [He-1] to conclude $f$ is an isomorphism.

Using the duality and a multiplicities argument as in [Hu-5] one may show

Proposition 2.8. Let $R$ be a local Gorenstein ring, $I=\left(a_{1}, \ldots, a_{n}\right)$ an ideal with $d(I)=d \leqslant 4$. If $H_{1}(\underline{a} ; R)$ and $H_{0}(\underline{a} ; R)$ are Cohen-Macaulay, then $H_{j}(\underline{a} ; R)$ are Cohen-Macaulay for all $j \geqslant 0$.

Corollary 2.9. Suppose $R$ is local Gorenstein and $I$ is an ideal generically a complete intersection with $R / I$ Cohen-Macaulay. Set $X=\operatorname{Spec}(R), Y=V(I)$. If $T_{2}^{X / Y}=0$, and $d(I) \leqslant 4$, then $Y$ is strongly Cohen-Macaulay in $X$ if and only if the conormal module, $I / I^{2}$, satisfies Serre's condition $S_{j}$ where $j=\operatorname{dim} Y-1$.

Proof. By the proposition above it is enough to prove $H_{1}$ is Cohen-Macaulay. Since $T_{2}^{X / Y}=0$, there is an exact sequence,

$$
0 \rightarrow H_{1} \rightarrow(R / I)^{n} \rightarrow I / I^{2} \rightarrow 0 .
$$

The result easily follows from standard depth considerations.

3. Main result. We refer the reader to the introduction for the definition of condition $G_{s}$. The purpose of this section is to prove

Theorem 3.1. Let $X=\operatorname{Spec}(R)$, where $R$ is a Cohen-Macaulay local ring, and let $Y=V(I)$ be a strongly Cohen-Macaulay closed subscheme of $X$. Assume $Y$ satisfies $G_{s}$. Let $a_{1}, \ldots, a_{s}$ be in $I$ such that $\left(a_{1}, \ldots, a_{s}\right)_{P}=I_{P}$ if ht $P \leqslant s$ and $P \in Y$. Set $Z=V(J)$ where $J=\left(a_{1}, \ldots, a_{s}: I\right)$, and we assume $\operatorname{codim} Z \geqslant s$. Let $W=$ $V\left(a_{1}, \ldots, a_{s}\right)$. Then:

(i) $Z$ is Cohen-Macaulay and $\operatorname{codim} Z=s$.

(ii) $W=Z \cup Y$.

(iii) $Z \cap Y$ is strongly Cohen-Macaulay in $Z$; in particular, $Z \cap Y$ is CohenMacaulay.

(iv) depth $R /\left(a_{1}, \ldots, a_{s}\right) \geqslant \operatorname{dim} R-s$.

We make a few remarks before beginning the proof of Theorem 3.1. The ideals $J$ arising in this fashion are called residual intersections by Artin and Nagata in their paper [A-N]. In that paper, Theorem 3.1 is stated [A-N, Theorem 2.1, p. 312] more generally; however this more general statement is incorrect as we shall show by a counterexample (Example 3.3). The statement (iii) of the theorem is necessary for the 
induction. Statements (i) and (iii) may be thought of as generalizations of Propositions 1.6 and 1.7 respectively. These propositions only dealt with the case $s=$ ht $I$, in which case $J$ is linked to $I$.

We will prove the theorem by induction on $s$, beginning with the case $s=k=$ ht $I$. In this case, (i) follows immediately from Proposition 1.6, while (iv) is also immediate as in this case $a_{1}, \ldots, a_{k}$ is a regular sequence. (Notice $\operatorname{ht}\left(a_{1}, \ldots, a_{k}\right)=$ ht $I$ as $\left(a_{1}, \ldots, a_{k}\right)_{P}=I_{P}$ for all primes $P$ minimal over $I$.) We show (ii). Let $\left(a_{1}, \ldots, a_{k}\right)$ $=q_{1} \cap \cdots \cap q_{m}$ be a primary decomposition of $\left(a_{1}, \ldots, a_{k}\right)$, and put $Q_{k}=\sqrt{q_{k}}$. To show $J \cap I=\left(a_{1}, \ldots, a_{k}\right)$ it suffices to prove $J \cap I \subset q_{i}$ for each $1 \leqslant i \leqslant m$. As $J I \subseteq\left(a_{1}, \ldots, a_{k}\right)$, it is enough to prove $J+I \nsubseteq Q_{i}$. However, if $I \subset Q_{i}$, then by assumption $I_{Q_{i}}=\left(a_{1}, \ldots, a_{k}\right)_{Q_{i}}$; so that $J_{Q_{i}}=\left(\left(a_{1}, \ldots, a_{k}\right)_{Q_{i}}: I_{Q_{i}}\right) \nsubseteq Q_{i}$. This shows both (ii) and the fact that $\operatorname{ht}(I+J) \geqslant k+1$. The assertion (iii) now follows from Proposition 1.7.

Henceforth we assume $s>k$ and assume the theorem is true for all values strictly less than $s$. We use a lemma which is a general position argument and is found in [A-N, Lemma 2.3, p. 312].

Lemma 3.2. Suppose $s>k$. The generators $a_{1}, \ldots, a_{s}$ may be chosen in such a way that $\left(a_{1}, \ldots, a_{s-1}\right)_{P}=I_{P}$ for all prime ideals $P \supseteq I$ such that ht $P \leqslant s-1$. Further, height $\left(a_{1}, \ldots, a_{s-1}: I\right)$ is at least $s-1$.

We now suppose we have chosen $a_{1}, \ldots, a_{s}$ as in Lemma 3.2. Set

$$
J_{1}=\left(a_{1}, \ldots, a_{s-1}: I\right) \text {. }
$$

By induction we may assume:

(1) $R / J_{1}$ is Cohen-Macaulay, ht $J_{1}=s-1$,

(2) $J_{1} \cap I=\left(a_{1}, \ldots, a_{s-1}\right)$,

(3) $I+J_{1} / J_{1}$ is strongly Cohen-Macaulay, and

(4) depth $R /\left(a_{1}, \ldots, a_{s-1}\right) \geqslant n-s+1$.

Let "' " denote the map from $R$ to $R / J_{1}$, and set $J^{*}=\left(\left(J_{1}, a_{s}\right): I\right)$. We first claim that $J^{*}=J$. It is clear that $J \subset J^{*}$ since $J I \subset\left(a_{1}, \ldots, a_{s}\right) \subseteq\left(J_{1}, a_{s}\right)$. To show the converse we need to prove $J^{*} I \subseteq\left(a_{1}, \ldots, a_{s}\right)$. By definition, $J^{*} I \subseteq\left(J_{1}, a_{s}\right)$ and so $J^{*} I \subseteq\left(J_{1}, a_{s}\right) \cap I$. Let $w \in J_{1}$ be such that $w+r a_{s} \in I$. Then $w \in I \cap J_{1}=$ $\left(a_{1}, \ldots, a_{s-1}\right)$. It follows that $\left(J_{1}, a_{s}\right) \cap I=\left(a_{1}, \ldots, a_{s}\right)$. Therefore, $J^{*} I \subseteq$ $\left(a_{1}, \ldots, a_{s}\right)$ and $J=J^{*}$.

We next prove that $\operatorname{ht}\left(J_{1}, a_{s}\right)=s$. If not, there is a prime $Q \supseteq\left(J_{1}, a_{s}\right)$, ht $Q=$ $s-1$. Then, as $I J \subseteq\left(a_{1}, \ldots, a_{s}\right) \subseteq\left(J_{1}, a_{s}\right) \subset Q$, either $I \subset Q$ or $J \subset Q$. However, as $\left(a_{1}, \ldots, a_{s-1}\right)_{p}=I_{p}$ at every prime of height $\leqslant s-1, I \nsubseteq Q$. Hence $J \subseteq Q$. However ht $J \geqslant s$. Thus $\operatorname{ht}\left(J_{1}, a_{s}\right)=s$. Since ht $J_{1}=s-1$ and $R / J_{1}$ is CohenMacaulay, $a_{s}^{\prime}$ is not a zero-divisor in $R / J_{1}$.

By the induction hypothesis, $I^{\prime}$ is strongly Cohen-Macaulay in $R^{\prime}$. By Proposition 1.6 it follows that $\left(J^{*}\right)^{\prime}=\left(a_{s}^{\prime}: I^{\prime}\right)$ satisfies the condition $R^{\prime} /\left(J^{*}\right)^{\prime}$ is Cohen-Macaulay. As $R^{\prime} /\left(J^{*}\right)^{\prime}=R / J^{*}=R / J$, we have shown (i).

We next show (ii). First, we will show ht $(I+J) \geqslant s+1$. This is clear from our assumption that $\left(a_{1}, \ldots, a_{s}\right)_{P}=I_{P}$ for every prime ideal $P \supseteq I$ such that ht $P \leqslant s$. 
Since $\left(J_{1}, a_{s}\right) \cap I=\left(a_{1}, \ldots, a_{s}\right)$, to show $J \cap I=\left(a_{1}, \ldots, a_{s}\right)$, it suffices to prove $J \cap I \subseteq\left(J_{1}, a_{s}\right)$. As $\operatorname{ht}(J+I) \geqslant s+1$ and $\left(J_{1}, a_{s}\right)$ is a height $s$ ideal such that $R /\left(J_{1}, a_{s}\right)$ is Cohen-Macaulay, it is enough to prove $J \cdot I \subseteq\left(J_{1}, a_{s}\right)$. However, $J=J^{*}=\left(\left(J_{1}, a_{s}\right): I\right)$.

To prove (iii) we use Proposition 1.7. We apply this proposition to the ideals $I^{\prime}$ and $\left(J^{*}\right)^{\prime}=J^{\prime}=\left(a_{s}^{\prime}: I^{\prime}\right)$ in $R^{\prime}$. We need to verify

(1) $J^{\prime} \cap I^{\prime}=\left(a_{s}^{\prime}\right)$, and

(2) $\operatorname{ht}\left(J^{\prime}+I^{\prime}\right) \geqslant 2$.

However, we have shown both of these conditions in the preceding argument. We conclude $I^{\prime}+J^{\prime} / J^{\prime}$ is strongly Cohen-Macaulay in $R / J^{\prime}$. Since $J_{1} \subset J$, this says $I+J / J$ is strongly Cohen-Macaulay in $R / J$, which proves (iii).

To prove (iv) we emulate [A-N]. Consider the exact sequences,

$$
\begin{gathered}
0 \rightarrow\left(a_{1}, \ldots, a_{s-1}\right) \rightarrow\left(a_{1}, \ldots, a_{s}\right) \rightarrow\left(a_{s}\right) /\left(a_{s}\right) \cap\left(a_{1}, \ldots, a_{s-1}\right) \rightarrow 0, \\
0 \rightarrow\left(a_{1}, \ldots, a_{s-1}\right) \rightarrow R \rightarrow R /\left(a_{1}, \ldots, a_{s-1}\right) \rightarrow 0 .
\end{gathered}
$$

As depth $R /\left(a_{1}, \ldots, a_{s-1}\right) \geqslant n-s+1$, where $n=\operatorname{dim} R$, from (4) we obtain

$$
\operatorname{depth}\left(a_{1}, \ldots, a_{s-1}\right) \geqslant n-s+2 \text {. }
$$

We next evaluate $\left(a_{s}\right) /\left(a_{s}\right) \cap\left(a_{1}, \ldots, a_{s-1}\right)$. Mapping $R$ onto this module we obtain

$$
R /\left(a_{1}, \ldots, a_{s-1}: a_{s}\right) \simeq\left(a_{s}\right) /\left(a_{s}\right) \cap\left(a_{1}, \ldots, a_{s-1}\right) .
$$

Notice $\left(a_{1}, \ldots, a_{s-1}: I\right)=J_{1} \subseteq\left(a_{1}, \ldots, a_{s-1}: a_{s}\right)$. In fact we claim these two ideals are equal. As $a_{s}\left(a_{1}, \ldots, a_{s-1}: a_{s}\right) \subseteq J_{1}$ and $a_{s}$ is not a zero-divisor on $R / J_{1}$, $\left(a_{1}, \ldots, a_{s-1}: a_{s}\right) \subseteq J_{1}$. We know depth $R / J_{1}=n-s+1$. It now follows from (3) that $\operatorname{depth}\left(a_{1}, \ldots, a_{s}\right) \geqslant n-s+1$. Consequently, $\operatorname{depth} R /\left(a_{1}, \ldots, a_{s}\right) \geqslant n-s$. This completes the proof of Theorem 3.1.

Finally, in this section we wish to give an example to show that the hypothesis of "strongly Cohen-Macaulay" is needed. This example provides a counterexample to Theorem 2.1 of $[\mathbf{A}-\mathbf{N}]$; however we note that the Corollary 2.2 in $[\mathbf{A}-\mathbf{N}]$ is correct since in this case the ideal in question is a complete intersection.

ExAmple 3.3. Let $X$ and $Y$ be generic two by two matrices over a field $k$; set $R=k[X, Y]$. (We adjoin the entries of $X$ and $Y$ to $k$.) Let $I=I_{1}(X Y)+I_{2}(X)+$ $I_{2}(Y)$. It is known that $R / I$ is Cohen-Macaulay of codimension 3. However, it is also known,

$$
I \cap I_{1}(X) \cap I_{1}(Y)=I_{1}(X Y)
$$

The ideal $I_{1}(X Y)$ is generated by four equations, and of course, $\operatorname{ht}\left(I_{1}(X) \cap I_{1}(Y)\right)$ $=4$. In addition $I$ satisfies $G_{\infty}$. If we could apply Theorem 3.1 to $I$, we could conclude $R /\left(I_{1}(X) \cap I_{1}(Y)\right)$ is Cohen-Macaulay. As this conclusion is obviously false, we have obtained the desired counterexample.

Observe that $v(I)=6=$ ht $I+3$. Hence Proposition 2.2 and Example 2.3 cannot be extended any further. 
4. Applications. We apply Theorem 3.1 to two different situations. The results of this section were already known. However the use of Theorem 3.1 gives very simple proofs, and what this author felt were very appealing proofs. These applications demonstrate some of the remarkable information which can be found in a strongly Cohen-Macaulay scheme. Furthermore Theorem 3.1 and the results here can be seen as a generalization of liaison.

We begin by recovering a classical result on determinantal varieties. We adopt the notation of the introduction, so that $D_{r, s}$ is the (affine) scheme whose closed points give maps from an $r$-dimensional space to an $s$-dimensional space having rank at most $r-1$. (Here $r \leqslant s$.) As noted before $D_{r, s}$ embeds in $\mathbf{A}_{k}^{r s}$ and it is easily seen that $D_{r, s}=\operatorname{Spec}\left(k\left[X_{i j}\right] / \sqrt{I_{r}}(X)\right)$. We wish to prove

THEOREM 4.1. The scheme $D_{r, s}$ is Cohen-Macaulay.

Proof. It is well known that it suffices to prove this statement at the origin. Accordingly, let $X=\operatorname{Spec}\left(k\left[X_{i j}\right]_{\left(X_{i j}\right)}\right)$, and $Y \subseteq X$ be the closed subscheme with $I(Y)=\sqrt{I_{r}}(X)$. We must prove $Y$ is Cohen-Macaulay.

We first extend the base $X$. Let $X^{\prime}$ be $\mathbf{A}_{k}^{s(s+1)}$ localized at the origin. We think of the coordinates of $\mathbf{A}_{k}^{s(s+1)}$ as an $s \times(s+1)$ matrix $A=\left(z_{i j}\right)$, with the right-most $r$ colums of $A$ giving the map of $X \rightarrow X^{\prime}$. (We set $B=$ right-most $r$ columns.) In $X^{\prime}$ we let $Z$ be the subscheme given by the ideal $I(Z)=I_{s}(A)$. As codimension $Z=2$, and $Z$ is perfect, by Proposition $0.3, Z$ is strongly Cohen-Macaulay in $X^{\prime}$. Further $Z$ satisfies $G_{\infty}$.

Let $Y^{\prime}=i(Y)$ where $i: X \rightarrow X^{\prime}$. As $i$ is a faithfully flat morphism, it is enough to prove $Y^{\prime}$ is Cohen-Macaulay. In fact we show $Y^{\prime}$ is a residual intersection of $Z$.

Let $a_{i}=$ the $s \times s$ matrix of $A$ determined by deleting the $i$ th column, and consider the ideal $\left(a_{1}, \ldots, a_{s-r+1}: I(Z)\right)=J$. Let $T=V(J)$. We will show $T=Y^{\prime}$.

By elementary linear algebra one can observe that $I(Z) \cdot I_{r}(B) \subseteq\left(a_{1}, \ldots, a_{s-r+1}\right)$. Hence, $I_{r}(B) \subseteq J$. On the other hand, since $I(Z)$ contains a nonzero-divisor modulo the ideal $\sqrt{I_{r}}(B)$ (which is prime and $=I\left(Y^{\prime}\right)$ ), we see that $J \subseteq \sqrt{I_{r}}(B)$. Therefore, $Y^{\prime}=T$, as topological spaces.

In particular, $\operatorname{codim} T=\operatorname{codim} Y^{\prime}=s-r+1$. It follows that $T$ is a residual intersection of $Z$ and consequently $T$ is Cohen-Macaulay. It remains to show $Y^{\prime}=T$ as schemes, i.e. that $\sqrt{I}_{r}(B)=\left(a_{1}, \ldots, a_{s-r+1}: I_{s}(A)\right)$. Since $T$ is Cohen-Macaulay, it has no embedded components. We only need to show $T$ is an integral scheme, and it suffices to do this at its unique generic point, $P=\sqrt{I}_{r}(B)$. However after localizing at $P,\left(I_{r}(B)\right)_{P}=\left(a_{1}, \ldots, a_{s-r+1}\right)_{P}=P_{P}$. Hence, $I_{r}(B)=\left(a_{1}, \ldots, a_{s-r+1}: I_{s}(A)\right)$ and so $Y^{\prime}=T$ is Cohen-Macaulay.

Next we wish to give an alternate proof of the theorem below of Simis and Vasconcelos by viewing the Rees algebra of an ideal $I$ as a residual intersection of $R / I$.

TheOREM 4.2 [S-V]. Let $R$ be a Cohen-Macaulay local ring and let $I$ be an ideal of $R$ such that $\operatorname{Spec}(R / I)=Y$ is strongly Cohen-Macaulay in $X=\operatorname{Spec}(R)$. Suppose $Y$ 
satisfies $G_{\infty}$. Then:

(1) $\operatorname{Sym}(I) \simeq \bigoplus_{n \geq 0} I^{n}$.

(2) $\oplus_{n \geqslant 0} I^{n}$ is Cohen-Macaulay.

(3) $\operatorname{Sym}\left(I / I^{2}\right)=\bigoplus_{n \geqslant 0} I^{n} / I^{n+1}$.

(4) $\bigoplus_{n \geq 0} I^{n} / I^{n+1}$ is Cohen-Macaulay.

In general, (1) holds if and only if (3) holds [Va], while (2) implies (4) [Hu-3].

We will actually only recover (1), (3) and (4). We wish to fix some notation. We let $T=\operatorname{Spec}\left(R\left[I t, t^{-1}\right]\right)$ where $R\left[I t, t^{-1}\right]$ is viewed as a subring of the ring $R\left[t, t^{-1}\right]$. (The variable $t$ is algebraically independent over $R$.) This ring is called the Rees algebra of $R$. If $G=\operatorname{Spec}\left(\bigoplus_{n \geqslant 0} I^{n} / I^{n+1}\right)$, the associated "canonical" scheme of $Y$, then $G=V\left(t^{-1}\right) \subseteq T$.

We also let $S=\operatorname{Spec}(\operatorname{Sym}(I))$, the scheme associated to the symmetric algebra of $I$, and let $W=\operatorname{Spec}\left(R\left[T_{1}, \ldots, T_{n}, U\right]\right)$ where $n=v(I) . S$ is a closed subscheme of $W$ defined by the vanishing of the equations $U$, and $\sum_{i=1}^{n} b_{i} T_{i}$ where $\sum_{i=1}^{n} b_{i} a_{i}=0$ (where $I=\left(a_{1}, \ldots, a_{n}\right)$ is a minimal generating set of $\left.I\right)$. Set $T^{\prime}=\operatorname{Spec}\left(R\left[T_{1}, \ldots, T_{n}, U\right] / J\right)$ where $J$ is the ideal generated by the equations $\sum_{i=1}^{n} b_{i} T_{i}\left(\sum_{i=1}^{n} b_{i} a_{i}=0\right)$, and the equations $U T_{i}-a_{i}$. There is an obvious surjection

$$
\frac{R\left[T_{1}, \ldots, T_{n}, U\right]}{J} \rightarrow R\left[I t, t^{-1}\right] \rightarrow 0
$$

by sending $T_{i}$ to $a_{i} t$ and $U$ to $t^{-1}$.

This gives a closed immersion of $T$ into $T^{\prime}$. Set

$$
Y^{\prime}=\operatorname{Spec}\left(R\left[T_{1}, \ldots, T_{n}, U\right] / R\left[T_{1}, \ldots, T_{n}, U\right](I, U)\right) .
$$

We will show that in general $T^{\prime}$ is a residual intersection of $Y^{\prime}$. Further, under the conditions of Theorem 3.1,T $=T^{\prime}$ and is Cohen-Macaulay. Finally, we interpret the equality $T=T^{\prime}$ as equivalent to the equality $\operatorname{Sym}(I)=\bigoplus_{n \geqslant 0} I^{n}$.

We proceed to show

Proposition 4.3. $T^{\prime}$ is a residual intersection of $Y^{\prime}$ if $\operatorname{codim} Y \geqslant 1$, and if $Y$ satisfies $G_{\infty}$.

Set $b_{i}=U T_{i}-a_{i}$. We will prove that $\left(\left(b_{1}, \ldots, b_{n}\right):(I, U)\right)=J$. First we prove $J$ is contained in the ideal on the left-hand side. This follows from the equations

$$
\left(\sum_{i=1}^{n} c_{i} T_{i}\right) U=\sum_{i=1}^{n} c_{i}\left(T_{i} U-a_{i}\right)
$$

and

$$
\left(\sum_{i=1}^{n} c_{i} T_{i}\right) a_{j}=\sum_{i=1}^{n} c_{i}\left(T_{i} a_{j}-a_{i} T_{j}\right),
$$

both of which are valid as $\sum_{i=1}^{n} c_{i} a_{i}=0$. Furthermore, $T_{i} a_{j}-a_{i} T_{j}=\left(U T_{i}-a_{i}\right) T_{j}-$ $\left(U T_{j}-a_{j}\right) T_{i}$. Conversely, suppose $F=F\left(T_{1}, \ldots, T_{n}, U\right) \in(\underline{b}:(I, U))$. Then there is an equation,

$$
F U=\sum_{i=1}^{n} G_{i} b_{i}=\sum_{i=1}^{n} G_{i}\left(U T_{i}-a_{i}\right) .
$$


Therefore,

$$
U\left(F-\sum_{i=1}^{n} G_{i} T_{i}\right)=-\sum_{i=1}^{n} a_{i} G_{i} .
$$

Write $G_{i}=G_{i}^{\prime}+U G_{i}^{\prime \prime}$ where $G_{i}^{\prime} \in R\left[T_{1}, \ldots, T_{n}\right]$. Equation (4) becomes

$$
U\left(F-\sum_{i=1}^{n} G_{i} T_{i}+\sum_{i=1}^{n} a_{i} G_{i}^{\prime \prime}\right)=\sum_{i=1}^{n} a_{i} G_{i}^{\prime} .
$$

Since $\sum_{i=1}^{n} a_{i} G_{i}^{\prime} \in R\left[T_{1}, \ldots, T_{n}\right]$, we see that

$$
\sum_{i=1}^{n} a_{i} G_{i}^{\prime}=0
$$

and

$$
F=\sum_{i=1}^{n} G_{i} T_{i}-\sum_{i=1}^{n} a_{i} G_{i}^{\prime \prime}=\sum_{i=1}^{n}\left(U T_{i}-a_{i}\right) G_{i}^{\prime \prime}+\sum_{i=1}^{n} G_{i}^{\prime} T_{i} .
$$

Therefore modulo $J, F=\sum_{i=1}^{n} G_{i}^{\prime} T_{i}$. From equation (5), $\sum_{i=1}^{n} a_{i} G_{i}^{\prime}=0$. As the map from $R$ to $R\left[T_{1}, \ldots, T_{n}\right]$ is faithfully flat, there exist relations $(1 \leqslant j \leqslant N), \sum_{i=1}^{n} a_{i} c_{i j}$ $=0$ in $R$, and polynomials $H_{j}$ in $R\left[T_{1}, \ldots, T_{n}\right]$ so that $G_{i}^{\prime}=\sum_{j=1}^{N} c_{i j} H_{j}$. Then

$$
F \equiv \sum_{i=1}^{n} T_{i} \sum_{j=1}^{N} c_{i j} H_{j} \equiv \sum_{j=1}^{N} H_{j}\left(\sum_{i=1}^{n} c_{i j} T_{i}\right) \equiv 0 \quad(\bmod J),
$$

since $\Sigma c_{i j} a_{i}=0$.

It follows that $J=\left(b_{1}, \ldots, b_{n}:(I, U)\right)$. Therefore $T^{\prime}$ is a residual intersection of $Y^{\prime}$ provided codim $T^{\prime}=n$. This follows from the lemma below.

Lemma 4.4. Suppose I satisfies $G_{\infty}$, and ht $I>0$. Then

$$
\operatorname{dim} \operatorname{Sym}(I)=\operatorname{dim} \bigoplus_{n \geq 0} I^{n}=\operatorname{dim} R+1 .
$$

Proof. The latter equality is well known. Let $I=\left(a_{1}, \ldots, a_{n}\right)$. There are surjections:

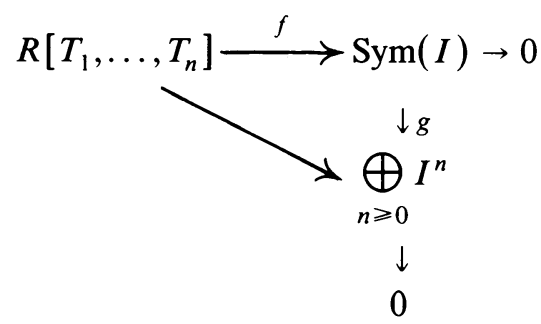

Set $A=\operatorname{ker} f, P=\operatorname{ker}(f \circ g) . P$ is a prime ideal. We need to show ht $A=$ ht $P$. Choose any minimal prime over $(A: P) \cap R$, and localize at it. It is enough to prove ht $A=$ ht $P$ in this localized ring. Thus we may assume $R$ is local with maximal ideal $m$, and $m^{N} P \subseteq A$, for some $N$. It follows that ht $A=\min ($ ht $P$, ht $m$ ); however ht $m=\operatorname{dim} R$, while ht $P=\operatorname{dim} R+v(I)-(\operatorname{dim} R+1)=v(I)-1$. Since $\operatorname{dim} R \geqslant v(I)$ because $I$ satisfies $G_{\infty}$, we obtain ht $A=$ ht $P$ as we required. 
It now easily follows that ht $J=n$ and consequently $T^{\prime}$ is a residual intersection of $Y^{\prime}$.

We are now able to give an alternate proof of Theorem 4.2(1), (3) and (4). Suppose our notations and assumptions are as in the statement of this theorem. By Proposition 4.3, $T^{\prime}$ is a residual intersection of $Y^{\prime}$. Since $Y^{\prime}$ is strongly CohenMacauiay and satisfies $G_{\infty}$, by Theorem 3.1 we conclude $T^{\prime}$ is Cohen-Macaulay. Further $(I, U)=I\left(Y^{\prime}\right)$ is not contained in the zero-divisors of $I\left(T^{\prime}\right)=J$. Therefore, $J:(I, U)=J$.

Let $A=I(T), A \subseteq R\left[T_{1}, \ldots, T_{n}, U\right]$. It is easily observed that $A$ is generated by the equations $U T_{i}-a_{i}$, plus all homogeneous polynomials $F\left(T_{1}, \ldots, T_{n}\right)$ with coefficients in $R$ such that $F\left(a_{1}, \ldots, a_{n}\right)=0$. In particular, if $\operatorname{deg} F=d$, then

$$
(I, U)^{d} F\left(T_{1}, \ldots, T_{n}\right) \subseteq\left(U T_{i}-a_{i}\right) .
$$

From (*) it follows that there exists an $M>0$ such that $A=\left(\left(U T_{i}-a_{i}\right):(I, U)^{M}\right)$. However, in this case $A=\left(J:(I, U)^{M-1}\right)=J$. Hence $T=T^{\prime}$ is Cohen-Macaulay. Since $\operatorname{Spec}\left(\operatorname{Sym}\left(I / I^{2}\right)\right)=V(U)$ in $T^{\prime}$ and $\operatorname{Spec}\left(\oplus I^{n} / I^{n+1}\right)=V(U)$ in $T$, we obtain statements (3) and (4) of Theorem 4.2. As we observed, Valla [Va] has shown that (3) implies statement (1). In general we do not know if statements (3) and (4) also imply (2); however we do not know of any counterexample either.

Finally, note that the applications in $\S \S 3$ and 4 of the paper of Artin and Nagata [A-N] now are valid with the hypothesis of strongly Cohen-Macaulay. We do not list these here, but refer the reader to the above paper.

5. A partial converse. Our purpose in this section is twofold. First we relate the residual intersection properties of Theorem 3.1 to the theory of $d$-sequences and approximation complexes, and secondly we use the latter theories to prove a converse to Theorem 3.1. An important ingredient is the use of the duality of the Koszul homology as we outlined in Proposition 2.7.

Suppose now that $X=\operatorname{Spec}(R)$, where $R$ is a Gorenstein local ring and assume $Y \subseteq X$ is a closed subscheme satisfying $G_{\infty}$. We will say $Y$ is residually CohenMacaulay in $X$ whenever $a_{1}, \ldots, a_{n} \in I(Y)$ such that $\operatorname{codim}\left(a_{1}, \ldots, a_{n}: I(Y)\right) \geqslant n$, then $Z=\operatorname{Spec}\left(R /\left(a_{1}, \ldots, a_{n}: I(Y)\right)\right)$ is Cohen-Macaulay of codimension $n$, $V\left(a_{1}, \ldots, a_{n}\right)=Z \cup Y$, and $Z \cap Y$ is Cohen-Macaulay of codimension $n+1$.

The main question of this section is the following: when is a residually CohenMacaulay scheme strongly Cohen-Macaulay? We will prove

THEOREM 5.1. Let $X=\operatorname{Spec}(R)$, where $R$ is a Gorenstein local ring and suppose $Y \subseteq X$ is a closed subscheme satisfying $G_{\infty}$ which is residually Cohen-Macaulay. Assume $c_{i}(Y) \geqslant i+2$ if $i \geqslant \operatorname{codim} Y+3$. Then $Y$ is strongly Cohen-Macaulay in $X$.

Our first step in proving Theorem 5.1 is to relate the condition of being residually Cohen-Macaulay to the property of being generated by a $d$-sequence.

Definition [Hu-4]. Let $x_{1}, \ldots, x_{n}$ be elements of a ring $R . x_{1}, \ldots, x_{n}$ are said to be a $d$-sequence if

(1) $x_{i} \notin\left(x_{1}, \ldots, \hat{x}_{i}, \ldots, x_{n}\right)$, and

(2) $x_{i}$ is not a zero-divisor on $R /\left(x_{0}, \ldots, x_{i-1}: x_{k}\right)$ for all $i \geqslant 0$, and all $k \geqslant i$. (Here we set $x_{0}=0$.) 
An ideal $J$ is said to be related to the $d$-sequence $x_{1}, \ldots, x_{n}$ if $J$ is either of the form $J=\left(x_{0}, \ldots, x_{i-1}: x_{i}\right)$ or $J=\left(x_{0}, \ldots, x_{i-1}: x_{i}\right)+\left(x_{1}, \ldots, x_{n}\right)$, where $1 \leqslant i \leqslant n$. The $d$-sequence is said to be a Cohen-Macaulay $d$-sequence (or a C-M $d$-sequence for short) if $R / J$ is Cohen-Macaulay for every related ideal $J$.

$D$-sequences were studied originally in $[\mathbf{H u}-4]$ to study the properties of powers of an ideal. It is known that if an ideal $I$ is generated by a $d$-sequence, then $\operatorname{Sym}(I) \simeq \bigoplus_{n \geq 0} I^{n}$. Further, if $I$ is generated by a Cohen-Macaulay $d$-sequence then $\operatorname{Sym}(I)$ is Cohen-Macaulay. An equivalent definition of a $d$-sequence is the condition $(1)+$ the condition $\left(2^{\prime}\right)$,

$$
\left(x_{0}, \ldots, x_{i-1}: x_{i}\right) \cap I=\left(x_{0}, \ldots, x_{i-1}\right)
$$

for all $1 \leqslant i \leqslant n\left(I=\left(x_{1}, \ldots, x_{n}\right)\right)$.

The following proposition is now apparent.

Proposition 5.2. Suppose $X=\operatorname{Spec}(R)$, where $R$ is a Cohen-Macaulay local ring and let $Y \subseteq X$ be a closed subscheme satisfying $G_{\infty}$ which is residually Cohen-Macaulay. Then $I(Y)=I$ can be generated by a Cohen-Macaulay $d$-sequence.

Proof. Since $Y$ satisfies $G_{\infty}$, we may choose minimal generators $a_{1}, \ldots, a_{n}$ of $I(Y)$ in such a way that $\left(a_{1}, \ldots, a_{s}\right)_{P}=I_{P}$ for all prime ideals $P \in Y$ such that ht $P \leqslant s$ (see Lemma 3.2). Then the assumption that $Y$ is residually Cohen-Macaulay forces $\left(a_{0}, \ldots, a_{i-1}: I\right) \cap I=\left(a_{0}, \ldots, a_{i-1}\right)$, and $R /\left(a_{0}, \ldots, a_{i-1}: I\right)$ to be CohenMacaulay. Further, $a_{i}$ is not a zero-divisor modulo $\left(a_{0}, \ldots, a_{i-1}: I\right)$. Since

$$
\left(a_{0}, \ldots, a_{i-1}\right) \subseteq\left(a_{0}, \ldots, a_{i-1}: I\right) \subseteq\left(a_{0}, \ldots, a_{i-1}: a_{i}\right),
$$

it follows that $\left(a_{0}, \ldots, a_{i-1}: I\right)=\left(a_{0}, \ldots, a_{i-1}: a_{i}\right)$. By definition, $a_{1}, \ldots, a_{n}$ are a Cohen-Macaulay $d$-sequence.

We need to make some remarks concerning $d$-sequences (see [Hu-4] for proofs).

REMARK 5.3. If $0 \leqslant i \leqslant n-1$, and $a_{1}, \ldots, a_{n}$ form a $d$-sequence, then the image of $a_{i+1}, \ldots, a_{n}$ in $R /\left(\left(a_{0}, \ldots, a_{i}\right): I\right)$ form a $d$-sequence $\left(I=\left(a_{1}, \ldots, a_{n}\right)\right)$.

REMARK 5.4. Any $d$-sequence satisfies $G_{\infty}$. In fact, any $d$-sequence is analytically independent. In particular, if $a_{1}, \ldots, a_{n}$ is a $d$-sequence in $R, n \leqslant \operatorname{dim} R$.

REMARK 5.5. If $I=\left(a_{1}, \ldots, a_{n}\right)$ where $a_{1}, \ldots, a_{n}$ is a $d$-sequence and grade $I=k$, then $a_{1}, \ldots, a_{k}$ form an $R$-sequence.

$D$-sequences are intimately connected with the study of the Tl-complex of [S-V], which played an essential role in the proofs in [S-V] of Theorem 4.2. Part of the relation between $d$-sequences and the T-complex was studied in [Hu-2], but in the works [HSV-1 and HSV-2], Herzog, Simis, and Vasconcelos brought to light the full connection.

In fact $d$-sequences play a role for the T-complex similar to the role regular sequences play for the Koszul complex. We recall the definition of the T-complex: let $\underline{x}=x_{1}, \ldots, x_{n}$ be a sequence of elements of $R$, and $L_{r, s}=K_{r} \otimes_{R} S_{s}$ be the double complex with differentials $\partial, \partial^{\prime}$ where $K_{r}=\Lambda^{r} F=r$ th exterior power of $F$, a free module with basis $e_{1}, \ldots, e_{n}$, and $S_{s}=\operatorname{Sym}_{s}(F)$. The differentials $\partial$ and $\partial^{\prime}$ are respectively obtained by viewing $L_{r, s}$ as either $K(\underline{x} ; R) \otimes \operatorname{Sym}(F)$, the Koszul maps determining $\partial$, or viewing the complex as the Koszul complex associated to the 
images of $e_{1}, \ldots, e_{n}$ in $\operatorname{Sym}_{1}(F)$. The two differentials commute, and the double complex gives rise to a complex $\Re=\mathfrak{R}(\underline{x} ; R)=\left\{H_{.} \otimes_{R} S, \partial^{\prime}\right\}$, where $H_{.}=$ homology of the Koszul complex of $x_{1}, \ldots, x_{n}$, and where $S=\operatorname{Sym}(F)$.

One of the main results of [HSV-2] is the following theorem.

THEOREM. Let $(R, m)$ be a local ring with infinite residue class field, and let $I$ be an ideal. Then the following are equivalent:

(1) $\Re(I ; R)$ is acyclic,

(2) I is generated by a d-sequence.

We should remark that the homology of $\mathfrak{N}$ is independent of the generating set of $I$. Moreover, in [HSV-2] the same results are shown to hold if we replace $R$ by an arbitrary finitely generated module $M$. Herzog, Simis, and Vasconcelos go on to relate the acyclicity of $\Re$ with the property of having a linear resolution. We refer the reader to [HSV-2] for details.

The key result in proving the converse of Theorem 3.1 is the next proposition which was almost proved in [Hu-2, Theorem 2.1].

Proposition 5.6. Let $R$ be a Cohen-Macaulay local ring and let $x_{1}, \ldots, x_{n}$ be $a$ Cohen-Macaulay $d$-sequence. Then,

$$
\text { depth } H_{i}(\underline{x} ; R) \geqslant \operatorname{dim} R-n+i
$$

for all $i, 1 \leqslant i \leqslant n-k, k=$ ht $I$.

Proof. We induct on $n$. Let $I=\left(x_{1}, \ldots, x_{n}\right)$.

Case 1. grade $I>0$. We show in this case that we may always reduce to a smaller $n$. Set $k=$ grade $I=$ ht $I$. We are assuming $k \geqslant 1$. By Remark 5.5, $x_{1}, \ldots, x_{k}$ form an $R$-sequence. Let “_” denote the map from $R$ to $R / R x_{1}$. By Lemma 1.4(1), there are exact sequences,

$$
0 \rightarrow H_{i}(I ; R) \rightarrow H_{i}(\bar{I} ; \bar{R}) \rightarrow H_{i-1}(I ; R) \rightarrow 0
$$

where the middle homology is taken with respect to $0, \bar{x}_{2}, \ldots, \bar{x}_{n}$. By $(1.1)$, the middle homology is isomorphic to

$$
H_{i}\left(\bar{x}_{2}, \ldots, \bar{x}_{n} ; R\right) \oplus H_{i-1}\left(\bar{x}_{2}, \ldots, \bar{x}_{n} ; R\right) .
$$

By induction on $n$, we may assume the depth of this module to be at least $\operatorname{dim} R-n+i-1$. We now induct on $j$ to show depth $H_{n-j}(I: R) \geqslant \operatorname{dim} R-n+$ $(n-j)$. The first nonzero homology is when $j=k$, and in this case, $H_{n-k}(I ; R) \simeq$ $\left(\left(x_{1}, \ldots, x_{k}\right): I\right) /\left(x_{1}, \ldots, x_{k}\right)$. By assumption, $R /\left(\left(x_{1}, \ldots, x_{k}\right): I\right)$ is Cohen-Macaulay, clearly of depth $R / I=\operatorname{dim} R-k$. It follows from the exact sequence

$$
0 \rightarrow\left(\left(x_{1}, \ldots, x_{k}\right): I\right) /\left(x_{1}, \ldots, x_{k}\right) \rightarrow R /\left(x_{1}, \ldots, x_{k}\right) \rightarrow R /\left(\left(x_{1}, \ldots, x_{k}\right): I\right) \rightarrow 0
$$

that depth $H_{n-k}(I ; R) \geqslant \operatorname{depth} R-k$. However, $R$ is Cohen-Macaulay so that $\operatorname{depth} R=\operatorname{dim} R$. Thus our assertion is verified for $j=k$.

Suppose $j>k$. Consider the exact sequence

$$
0 \rightarrow H_{n-j+1}(I ; R) \rightarrow H_{n-j+1}(\bar{I} ; \bar{R}) \rightarrow H_{n-j}(I ; R) \rightarrow 0 .
$$


By our induction on $n$, depth $H_{n-j+1}(\bar{I} ; \bar{R}) \geqslant \operatorname{dim} R-j+1$. By our induction on $j$, depth $H_{n-j+1}(I ; R) \geqslant \operatorname{dim} R-j+1$. It now follows depth $H_{n-j}(I ; R) \geqslant$ $\operatorname{dim} R-j$.

Case 2. grade $I=0$. Let "_" denote the homomorphism from $R$ to $R /(0: I)$. We know $(0: I) \cap I=(0)$. By Lemma $1.4(2)$ there are exact sequences

$$
0 \rightarrow \bigoplus(0: I) \rightarrow H_{i}(I ; R) \rightarrow H_{i}(\bar{I}, \bar{R}) \rightarrow 0 .
$$

Since we are assuming $R /(0: I)$ is Cohen-Macaulay (since $(0: I)$ is a related ideal), it follows that $(0: I)$ is also Cohen-Macaulay. As $(0: I)=\left(0: x_{1}\right)=\left(0: x_{1}^{2}\right)$, grade $\bar{I} \geqslant 1$, and we may apply Case 1 to conclude depth $H_{i}(\bar{I}, \bar{R}) \geqslant \operatorname{dim} \bar{R}-n+i$. Since $\operatorname{depth}(0: I)=\operatorname{depth} R / I=\operatorname{depth} I$, it easily follows from the exact sequence above that depth $H_{i}(I ; R) \geqslant \operatorname{dim} R-n+i$. (Note that the images of $x_{1}, \ldots, x_{n}$ form a $d$-sequence in $R /(0: I)$ by Remark 5.3.)

Corollary 5.7. Let $R$ be a Gorenstein local ring and let $x_{1}, \ldots, x_{n}$ be a $C-M$ $d$-sequence. Set $I=\left(x_{1}, \ldots, x_{n}\right)$, set $Y=\operatorname{Spec}(R / I)$. Suppose $c_{i}(Y) \geqslant i+1$ if $i \geqslant$ codim $Y+2$. Then $H_{i}(\underline{x} ; R)$ are reflexive for all $0 \leqslant i \leqslant d(I)$.

Proof. First observe that the condition, " $H_{i}(\underline{x} ; R)$ is reflexive for all $i, 0 \leqslant i \leqslant$ $d(I)$ " is independent of the generating set chosen. We need to prove $H_{i}(\underline{x} ; R)_{P}$ is isomorphic to $H_{i}(\underline{x} ; R)_{P}^{* *}$ if ht $P / I \leqslant 1$, and further show $\operatorname{depth}\left(H_{i}(\underline{x} ; R)\right)_{P} \geqslant 2$ if ht $P / I \geqslant 2$. Suppose ht $P / I \leqslant 1$. In this case since $Y$ satisfies $G_{\infty}, v\left(I_{P}\right) \leqslant$ ht $I+1$. As we may choose whatever generating set we want to show that $H_{i}(\underline{x} ; R)_{P}$ are reflexive, we choose a minimal generating set of $\underline{x}^{\prime}$ of $I_{P}$. Then, $H_{i}\left(\underline{x}^{\prime} ; R_{P}\right)=0$ for $i \geqslant 2$ while $H_{1}\left(\underline{x}^{\prime} ; R_{P}\right)=0$, or is the canonical module of $R / I$. It follows that $\left(H_{i}(\underline{x} ; R)\right)_{P}$ is reflexive if ht $P / I \leqslant 1$.

Assume ht $P / I \geqslant 2$, and localize at $P$. We assume $R$ is local with maximal ideal $P$. We need to show depth $H_{i}(\underline{x} ; R) \geqslant 2$ for all $1 \leqslant i \leqslant d(I)$. We use Proposition 5.6. If $i \geqslant 2$, then since

$$
\text { depth } H_{i}(\underline{x} ; R) \geqslant \operatorname{dim} R-v(I)+i \geqslant 2
$$

there is nothing to show. If $i=1$, it suffices to show $\operatorname{dim} R>v(I)$. If not, $v(I)=\operatorname{dim} R$. Set $n=v(I)$, and consider $c_{n}(Y)$. Since $\operatorname{dim} R \geqslant \operatorname{dim} R / I+2$, our assumption tells us that $c_{n}(Y) \geqslant n+1$. As $n=\operatorname{dim} R$, this is a contradiction. Therefore $\operatorname{dim} R>v(I)$, and depth $H_{1}(\underline{x} ; R) \geqslant 2$.

In particular, under the conditions of Corollary 5.7, there is a pairing,

$$
H_{i}(\underline{x} ; R) \times H_{d-i}(\underline{x} ; R) \rightarrow H_{d}(\underline{x} ; R)=K_{R / I} .
$$

Here $d=d(I)$ (see Proposition 2.7).

We will use this duality of the Koszul homology along with Proposition 5.6 to prove under fairly weak conditions that the Koszul homology must be CohenMacaulay.

The key to this attempt is the following lemma. Although it is essentially found in [H-0 and FFGR, Corollary 2.6], it does not seem to be well known in the form below. However it is extremely useful. 
LemMa 5.8. Let $R$ be a local Cohen-Macaulay ring with canonical module $K_{R}$. Let "“" denote $\operatorname{Hom}\left(-, K_{R}\right)$. Suppose $M_{p}$ is Cohen-Macaulay if ht $p \leqslant 2$ and assume

$$
\operatorname{depth} M_{p}+\operatorname{depth}\left(M^{\nu}\right)_{p} \geqslant \operatorname{dim} R_{p}+2
$$

for all prime ideals $p$ of $R$ of height at least three with $M_{p} \neq 0$. Then $M$ is a maximal Cohen-Macaulay module.

The proof is a straightforward use of duality, but for completeness we give the proof here.

Since $\operatorname{Hom}_{R}\left(M, K_{R}\right)_{p}=\operatorname{Hom}\left(M_{p},\left(K_{R}\right)_{p}\right)=\operatorname{Hom}_{R_{p}}\left(M_{p}, K_{R_{p}}\right)$, the assumptions and statement of the lemma remain valid under localization. We may therefore assume that $M_{p}$ is Cohen-Macaulay if $p \neq m$, the maximal ideal of $R$. Further we may assume $\operatorname{dim} R \geqslant 3$ and $M_{p} \neq 0$.

Let $F$. be a free resolution of $M$, and consider the complex $F_{\text {. }}$. The cohomology of $F_{\text {. is }} \operatorname{Ext}_{R}^{i}\left(M, K_{R}\right)$, which in turn is dual to $H_{m}^{d-i}(M)$, if $d=\operatorname{dim} R$.

We wish to prove $H_{m}^{i}(M)=0$ for $i<d$. Set $k=\operatorname{depth} M$. Then $H_{m}^{i}(M)=0$ for $i<k$. We have assumed

$$
\operatorname{depth} M^{\check{2}} \geqslant d+2-k \text {. }
$$

In addition $\operatorname{Ext}_{R}^{i}\left(M, K_{R}\right)$ has finite length if $i \geqslant 1$, since $M_{p}$ is Cohen-Macaulay if $p \neq m\left(\right.$ or $\left.M_{p}=0\right)$. Consider the complex

$$
0 \rightarrow M^{\check{ }} \rightarrow F_{0}^{\check{\nu}} \rightarrow F_{1}^{\check{\nu}} \rightarrow \cdots \rightarrow F_{j}^{\check{\nu}} \rightarrow \cdots .
$$

We are in a position to apply the lemma of acyclicity of Peskine and Szpiro [P-S-2]. As depth $M \geqslant d+2-k$, and depth $F_{i} \geqslant d$ for all $i$, we may conclude the complex (1) is exact up to $j=d-k+1$, i.e. that $\operatorname{Ext}^{i}\left(M, K_{R}\right)=0$ if $1 \leqslant i \leqslant d-k$. In this case, $H_{m}^{d-i}(M)=0$ for $1 \leqslant i \leqslant d-k$, so that $H_{m}^{j}(M)=0$ for $k \leqslant j<d$. It follows that $M$ is Cohen-Macaulay.

We observe that the well-known criterion of Hartshorne and Ogus follows at once from this lemma.

Proposition [H-O]. Let $R$ be a local ring, quotient of a Gorenstein ring and assume $R$ is factorial. If

$$
\operatorname{depth} R_{p} \geqslant \frac{1}{2}\left(\operatorname{dim} R_{p}\right)+1
$$

for all $p \in \operatorname{Spec}(R)$, then $R$ is Cohen-Macaulay.

Proof. We may assume $R$ is a quotient of a Gorenstein local ring $S$ with $\operatorname{dim} S=\operatorname{dim} R$. By Lemma 5.8 if

$$
\operatorname{depth} R_{p}+\operatorname{depth}\left(K_{R}\right)_{p} \geqslant \operatorname{dim} R_{p}+2
$$

for all $p \in \operatorname{Spec}(R)$ of height at least 3 , then $R$ is Cohen-Macaulay. Since $R$ is factorial, it is well known that $K_{R}=R$. Therefore (2) reads

$$
\text { depth } R_{p} \geqslant \frac{1}{2}\left(\operatorname{dim} R_{p}\right)+1 \text {. }
$$

TheOREM 5.9. Let $R$ be a Gorenstein local ring, and let $x_{1}, \ldots, x_{n}$ be a CohenMacaulay d-sequence. Set $I=\left(x_{1}, \ldots, x_{n}\right), Y=\operatorname{Spec}(R / I)$. Suppose $c_{i}(Y) \geqslant i+2$ if $i \geqslant \operatorname{codim} Y+3$. Then $Y$ is strongly Cohen-Macaulay in $X=\operatorname{Spec}(R)$. 
Proof. We induct on $\operatorname{dim} R$. We may assume that $H_{i}(\underline{x} ; R)_{p}$ is Cohen-Macaulay if $p \neq m$, the maximal ideal of $R$. Furthermore we may assume $\operatorname{dim} R / I \geqslant 3$, since if $\operatorname{dim} R / I \leqslant 2$, then Example 2.3 and Proposition 2.2 show that $Y$ will be strongly Cohen-Macaulay. By Corollary 5.7, we may assume (with $d=d(I)$ )

$$
\begin{aligned}
& H_{i}(\underline{x} ; R)=\operatorname{Hom}\left(H_{d-i}(\underline{x} ; R), H_{d}(\underline{x} ; R)\right), \\
& H_{d-i}(\underline{x} ; R)=\operatorname{Hom}\left(H_{i}(\underline{x} ; R), H_{d}(\underline{x} ; R)\right) .
\end{aligned}
$$

By using Lemma 5.8, it is enough to prove

$$
\text { depth } H_{i}(\underline{x} ; R)+\operatorname{depth} H_{d-i}(\underline{x} ; R) \geqslant \operatorname{dim} R / I+2
$$

since $H_{d}(\underline{x} ; R)=K_{R / I}$. However, applying Proposition 5.6 shows that

$$
\begin{aligned}
\operatorname{depth} H_{i}(\underline{x} ; R) & +\operatorname{depth} H_{d-i}(\underline{x} ; R) \\
\geqslant & \operatorname{dim} R-v(I)+i+\operatorname{dim} R-v(I)+d-i \\
= & 2 \operatorname{dim} R-2 v(I)+d .
\end{aligned}
$$

It remains to show

$$
2 \operatorname{dim} R-2 v(I)+(v(I)-\text { ht } I) \geqslant \operatorname{dim} R-\text { ht } I+2,
$$

i.e. that $\operatorname{dim} R \geqslant v(I)+2$. Set $n=v(I)$. As we may assume $d \geqslant 3$, by assumption $c_{n}(Y) \geqslant n+2$. In particular, $\operatorname{dim} R \geqslant n+2$ which finishes the proof of the theorem.

We may now prove (and restate)

Theorem 5.1 (Partial Converse to Theorem 3.1). Let $X=\operatorname{Spec}(R)$, where $R$ is a Gorenstein local ring and suppose $Y \subseteq X$ is a closed subscheme satisfying $G_{\infty}$ which is residually Cohen-Macaulay. Assume $c_{i}(Y) \geqslant i+2$ if $i \geqslant \operatorname{codim} Y+3$. Then $Y$ is strongly Cohen-Macaulay in $X$.

Proof. By Proposition 5.2, $I=I(Y)$ can be generated by a Cohen-Macaulay $d$ sequence. We now simply apply Theorem 5.9.

We close with an example to point out the Gorenstein condition on the base ring is necessary.

EXAmple 5.10. Let $R=k\left[X_{i j}\right] / I_{2}(X)$, where $X=\left(X_{i j}\right)$ is a $2 \times 3$ matrix. Denote the image of $X_{i j}$ in $R$ by $x_{i j}$, and let $I=\left(x_{11}, x_{12}, x_{13}\right)$. It is easily observed that $I$ is generated by a Cohen-Macaulay $d$-sequence. Further, $C_{2}(R / I)=\{m\}$, where $m=$ $\left(x_{i j}\right)$. Thus,

$$
c_{2}(R / I)=c_{2}(R / I)=4, \quad c_{i}(R / I)=\infty \quad \text { for } i \geqslant 3 .
$$

In particular, $c_{i}(Y) \geqslant i+2$ if $i \geqslant \operatorname{codim} Y+3=4$. Also $R / I$ satisfies $G_{\infty}$ (as it must since $I$ is generated by a $d$-sequence). However, one can compute that depth $H_{1}\left(x_{11}, x_{12}, x_{13} ; R\right)=2$.

Therefore $H_{1}\left(x_{11}, x_{12}, x_{13} ; R\right)$ is not Cohen-Macaulay. However, $R$ is not Gorenstein. This example also shows that Example 2.3 cannot be improved by removing the assumption that $R$ be Gorenstein. Notice $v(I)=3=$ ht $I+2$. 


\section{REFERENCES}

[A-N] M. Artin and M. Nagata, Residual intersections in Cohen-Macaulay rings, J. Math. Kyoto Univ. 12 (1972), 307-323.

[A-H] L. Avramov and J. Herzog, The Koszul algebra of a codimension 2 embedding, Math Z. 175 (1980), 249-260.

[B-E] D. Buchsbaum and D. Eisenbud, What makes a complex exact? J. Algebra 25 (1973), 259-268.

[FFGR] R. Fossum, H. Foxby, P. Griffith and I. Reiten, Minimal injective resolutions with applications to dualizing modules and Gorenstein modules, Inst. Hautes Études Sci. Publ. Math. 45 (1976).

[G-L] T. H. Gulliksen and G. Levin, Homology of local rings, Queen's Paper in Pure and Appl. Math., No. 20, Queen's Univ. Press, Kingston, 1969.

[H-O] R. Harthshorne and A. Ogus, On the factoriality of local rings of small embedding codimension, Comm. Algebra 1 (1974), 415-437.

[He-1] J. Herzog, Komplexe, Auflosungen und Dualitat in der lokalen Algebra, Habilitationsschriff, Regensburg Universität, 1974.

[He-2] , Certain complexes associated with a sequence and a matrix, Manuscripta Math. 12 (1974), 217-248.

[HSV-1] J. Herzog, A. Simis and W. V. Vasconcelos, Approximation complexes of blowing-up rings. II, J. Algebra (to appear).

[HSV-2] __ Approximation complexes of blowing-up rings. II, J. Algebra (to appear)

[Hu-1] C. Huneke, Linkage and the Koszul homology of ideals, Amer. J. Math. 104 (1982), 1043-1062.

[Hu-2] __ The Koszul homology of an ideal, Advances in Math. (to appear).

[Hu-3] __ On the associated graded ring of an ideal, Illinois J. Math. 26 (1982), 121-137.

[Hu-4] _ The theory of $d$-sequences and powers of ideals, Advances in Math. (to appear).

[Hu-5] _ The invariants of liaison, Proc. Third Midwest Algebraic Geom. Conf., Lecture Notes in Math., Springer-Verlag, Berlin and New York (to appear).

[K] S. Kleiman, Multiple-point formulas. I: Iteration, Acta Math. 147 (1981), 13-49.

[Ku] M. Kühl, Über die symmetrische Algebra eines Ideals, dissertation, Essen, 1981.

[L] S. Lichtenbaum, On the vanishing of Tor in regular local rings, Illinois J. Math. 10 (1966), 220-226.

[M] S. Mac Lane, Homology, Springer-Verlag, Berlin, Heidelberg and New York, 1963.

[P-S-1] C. Peskine and L. Szpiro, Liaisons des variétés algébriques, Invent. Math. 26 (1974), 271-302. [P-S-2] , Dimension projective finie et cohomologie locale, Inst. Hautes Études Sci. Publ. Math. 42 (1973)

[S-V] A. Simis and W. Vasconcelos, The syzygies of the conormal module, Amer. J. Math. 103 (1980), 203-224.

[Va] G. Valla, On the symmetric and Rees algebra of an ideal, Manuscripta Math. 30 (1980), 239-255.

[V] W. Vasconcelos, On the homology of $I / I^{2}$, Comm. Algebra 6 (17), (1978), 1801-1809.

[W] J. Watanabe, A note on Gorenstein rings of embedding codimension 3, Nagoya Math. J. 50 (1973), 333-341.

DePartment of Mathematics, University of Illinois, Urbana, Illinois 61801 